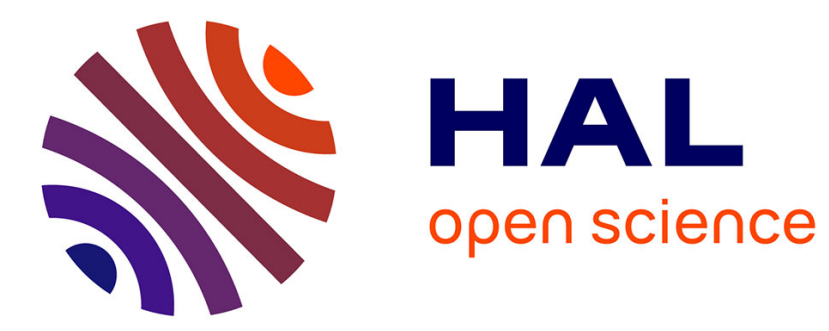

\title{
A Continuous Exact 10 penalty (CEL0) for least squares regularized problem
}

\author{
Emmanuel Soubies, Laure Blanc-Féraud, Gilles Aubert
}

\section{To cite this version:}

Emmanuel Soubies, Laure Blanc-Féraud, Gilles Aubert. A Continuous Exact 10 penalty (CEL0) for least squares regularized problem. SIAM Journal on Imaging Sciences, 2015, 8 (3), pp. 1607-1639 (33 p.). 10.1137/151003714. hal-01102492v2

\section{HAL Id: hal-01102492 https://inria.hal.science/hal-01102492v2}

Submitted on 22 May 2015

HAL is a multi-disciplinary open access archive for the deposit and dissemination of scientific research documents, whether they are published or not. The documents may come from teaching and research institutions in France or abroad, or from public or private research centers.
L'archive ouverte pluridisciplinaire HAL, est destinée au dépôt et à la diffusion de documents scientifiques de niveau recherche, publiés ou non, émanant des établissements d'enseignement et de recherche français ou étrangers, des laboratoires publics ou privés. 


\title{
A Continuous Exact $\ell_{0}$ penalty (CELO) for least squares regularized problem.
}

\author{
Emmanuel Soubies \\ Laure Blanc-Féraud ${ }^{\dagger}$ \\ Gilles Aubert $\ddagger$
}

May 20, 2015

\begin{abstract}
Within the framework of the $\ell_{0}$ regularized least squares problem, we focus, in this paper, on nonconvex continuous penalties approximating the $\ell_{0}$-norm. Such penalties are known to better promote sparsity than the $\ell_{1}$ convex relaxation. Based on some results in one dimension and in the case of orthogonal matrices, we propose the Continuous Exact $\ell_{0}$ penalty (CEL0) leading to a tight continuous relaxation of the $\ell_{2}-\ell_{0}$ problem. The global minimizers of the CEL0 functional contain the global minimizers of $\ell_{2}-\ell_{0}$ and from each global minimizer of CEL0 one can easily identify a global minimizer of $\ell_{2}-\ell_{0}$. We also demonstrate that from each local minimizer of the CEL0 functional, a local minimizer of $\ell_{2}-\ell_{0}$ is easy to obtain. Moreover, some strict local minimizers of the initial functional are eliminated with the proposed tight relaxation. Then solving the initial $\ell_{2}-\ell_{0}$ problem is equivalent, in a sense, to solve it by replacing the $\ell_{0}$-norm with the CEL0 penalty which provides better properties for the objective function in terms of minimization, such as the continuity and the convexity with respect to each direction of the standard $\mathbb{R}^{N}$ basis, although the problem remains nonconvex. Finally, recent nonsmooth nonconvex algorithms are used to address this relaxed problem within a macro algorithm ensuring the convergence to a critical point of the relaxed functional which is also a (local) optimum of the initial problem.
\end{abstract}

Key words inverse problems, $\ell_{0}$ regularization, sparse modelling, underdetermined linear systems, global minimizers, local minimizers, minimizers equivalence, Continuous Exact $\ell_{0}$ penalty, nonconvex nonsmooth penalty

\section{Introduction}

In many applications such as coding (to reduce data storage), compressed sensing (to recover a signal from fewer measurements), source separation, variable selection, image decomposition and many others, one aims to compute a sparse solution of an underdetermined linear system of equations. In other words these problems search for an approximation of a signal as a linear combination of redundant dictionary atoms also known as synthesis approach. The underlying idea relies on the existence of a representation of the unknown signal involving only few atoms of the dictionary. This can be modeled using sparsity constraints or penalties. The problem reads as a least-squares loss function $\|A \mathrm{x}-d\|^{2}$

\footnotetext{
${ }^{\dagger}$ Université de Nice Sophia Antipolis, CNRS, Laboratoire I3S UMR 7271, 06903 Sophia Antipolis, France (soubies@i3s.unice.fr, blancf@i3s.unice.fr)

$\ddagger$ Université de Nice Sophia Antipolis, CNRS, Laboratoire J.A. Dieudonné UMR 7351,06100 Nice, France (gilles.aubert@unice.fr)
} 
defined from a matrix $A \in \mathbb{R}^{M \times N}$ and data $d \in \mathbb{R}^{M}$ plus a sparsity prior usually provided by the $\ell_{0}$-norm ${ }^{1}$, also known as " $0-1$ " function, and defined by

$$
\|\mathrm{x}\|_{0}=\#\left\{\mathrm{x}_{i}, i=1, \cdots, N: \mathrm{x}_{i} \neq 0\right\},
$$

where \# denotes cardinality. This problem can be stated under different formulations. The constrained forms are used when a prior is known either on the sparsity of the solution or on the noise variance. When we seek a solution $\hat{\mathrm{x}}$ under the constraint $\|\hat{\mathrm{x}}\|_{0} \leq k \in \mathbb{N}$, the problem is usually formulated as follows,

$$
\hat{\mathrm{x}}=\underset{\mathrm{x} \in \mathbb{R}^{N}}{\arg \min }\|A \mathrm{x}-d\|_{2}^{2} \text { subject to }\|\mathrm{x}\|_{0} \leq k .
$$

Otherwise, a knowledge about the variance of the noise leads to the formulation

$$
\hat{\mathrm{x}}=\underset{\mathrm{x} \in \mathbb{R}^{N}}{\arg \min }\|\mathrm{x}\|_{0} \text { subject to }\|A \mathrm{x}-d\|_{2}^{2} \leq \epsilon,
$$

where $\epsilon>0$ is determined with respect to the statistic of the data $d \in \mathbb{R}^{M}$. Finally, when no prior about the noise or the sparsity of the solution is known (i.e. we do not know $k$ and $\epsilon$ ) the penalized form

$$
\hat{\mathrm{x}} \in \underset{\mathrm{x} \in \mathbb{R}^{N}}{\arg \min } \mathrm{G}_{\ell_{0}}(\mathrm{x}):=\frac{1}{2}\|A \mathrm{x}-d\|_{2}^{2}+\lambda\|\mathrm{x}\|_{0},
$$

is used. Here, $\lambda>0$ is an hyperparameter characterizing the trade-off between data fidelity and sparsity. These problems are well known to be NP-Hard [14, 29]. Note that in general, because of the nonconvexity, they are not equivalent [33]. In the work of Geman and Geman [21], the $\ell_{0}$-norm was already used as a regularizer of MAP energies for image restoration. The "0-1" functional was combined with a discrete derivative operator leading to the Potts prior model. Since this pioneering work, numerous methods/principles have been proposed to find a good approximate solution of these problems. We can group them into four categories.

Convex relaxation One common alternative is the convex relaxation of this combinatorial problem. It consists in replacing the $\ell_{0}$-norm by the convex $\ell_{1}$-norm to take advantage of well-known algorithms in convex optimization. The Basis Pursuit (BP) principle or its De-Noising (BPDN) adaptation, proposed by Chen et al [9], deals with the $\ell_{1}$ relaxation of problems (1.3) and (1.4) using linear and quadratic programming algorithms (e.g. simplex, active set or interior-point methods). Iterative thresholding algorithms such as ISTA (Daubechies et al [13]) and its accelerated version FISTA (Beck et al [2]), belonging to the family of forward-backward algorithms [12], are now well-known to be very efficient for such $\ell_{1}$ relaxed problems. The fact that this convex relaxation provides sparse solutions comes from the singularity of the $\ell_{1}$-norm at zero [17]. For illustration, one can see that when the matrix $A$ is orthogonal, solving the relaxed $\ell_{1}$ problem leads to a soft-thresholding which sets small coefficients to zero. In fact, a necessary and sufficient condition to obtain a thresholding rule as estimator (in the case of orthogonal matrices) is that the associated penalty be singular at the origin [17]. Sufficient conditions have been provided by several authors $[16,7,41]$ on the matrix $A$ under which it is shown that sufficiently sparse signals can be exactly recovered from $\ell_{1}$ minimization. These conditions are usually too restrictive for practical applications.

Greedy algorithms Another class of algorithms dedicated to these problems are the greedy methods based on the following idea: starting from a null solution, the most relevant components (with respect to some criteria) are added iteratively one by one. The Matching Pursuit (MP), introduced in [26]

\footnotetext{
${ }^{1}$ Note that even if it is not a norm since the absolute homogeneity is not verified we will refer to it as $\ell_{0}$-norm.
} 
by Mallat et al, is an iterative algorithm selecting one atom per iterate that matches at best the residual - maximizing the correlation - until the desired accuracy is reached. The residual is proved to converge exponentially to zero. A revision of this algorithm, the Orthogonal Matching Pursuit (OMP), have been proposed by Pati et al [35]. OMP improves the convergence performances of MP by adding, at each iterate, a least-squares minimization allowing to get the best approximation over the selected atoms. We can also cite the Orthogonal Least Squares (OLS) introduced previously by Chen et al [10] and similar to OMP. Tropp [41] gives a theoretical result which states that under a sufficient incoherence assumption, OMP recovers the sparsest representation of the input signal. He showed that this result also stand for BP. However some authors (see [9, §2.3.2] and references therein) have provided examples where these greedy algorithms fail to provide sparse solutions. An adaptation of OMP and OLS has been proposed as Single Best Replacement (SBR) by Soussen et al [39]. At each iteration an atom can be selected or de-selected. Then a wrong selected atom in the first few iterates, which is a major drawback of classical greedy algorithms, can be latter removed. A survey of greedy algorithms can be found in [40].

Graduated Non Convexity (GNC) algorithms GNC approaches have been initially introduced by Blake and Zisserman in [3] for the minimization of nonconvex functions. The main idea is to minimize a sequence of functions starting with a convex one and introducing progressively the nonconvexity. In the context of image segmentation and coding, Leclerc [25] proposed a GNC based algorithm to approach a local minima of a $\ell_{0}$-penalized functional. A generalization of GNC approaches has been proposed later by Nikolova [30] for Maximum A Posteriori (MAP) energies involving Markovian prior for image reconstruction. This work deals with a range of Potential Functions (PF) including the $\ell_{0}$ norm and provides an interesting study on the way to construct and initialize the relaxed sequence of functionals. More recently, Mohimani et al [28] used this principle in the context of sparse approximation where they proposed the SL0 (Smoothed $\ell_{0}$ ) algorithm to deal with the constrained problem (1.3). They proved the convergence to the sparsest solution under some conditions on $\|A\|[27]$. Also, it is worth noting that Robini et al $[37,38]$ did important progress in terms of global minimization by combining Simulated Annealing and GNC to take benefit from the advantages of these two approaches.

Continuous nonsmooth nonconvex penalties This last point is more a principle than a method although algorithms have also been proposed in this context. Replacing the noncontinous nonconvex $\ell_{0}$-norm by a nonsmooth nonconvex but continuous penalty has been widely investigated in the statistic community in the context of variable selection. The main motivation was to overstep the bias introduced by the $\ell_{1}$ penalty on large coefficients $[17,43]$. Among the variety of such penalties which have been proposed, we can mention the NonNegative Garrote [6], the Log-Sum penalty [8] or the Capped- $\ell_{1}$ [36]. Also, Fan and Li [17] defined necessary conditions to obtain a "good" penalty function (unbiasedness, continuity in data, sparsity) and propose the Smoothly Clipped Absolute Deviation (SCAD) penalty [17]. In the same spirit, Zhang [42] introduced the notion of sparse convexity to compare penalties and proposed Minimax Concave Penalty (MCP). Fourcart et Lai [18] investigated the use of $\ell_{p}$-norms $(0<p<1)$ instead of the $\ell_{0}$ one (see also references in [18]) and an interesting work on the equivalence between minimal $\ell_{0}$ and $\ell_{p}(0<p \leq 1)$ solutions of linear systems of equations (equality or inequality) can be found in [19]. Recently, Le Thi et al [24] proposed an exact reformulation of $\ell_{0}$ regularized problems as DC programs. They also show that some nonconvex approximations (e.g. Capped- $\ell_{1}$ ) are equivalent to their reformulation. Finally the Iterative Hard Thresholding (IHT) is a well known algorithm which deals directly with problems (1.2) or (1.4). It has been initially introduced by Blumensath and Davies in [4] under two variants dedicated respectively to problem (1.4) and (1.2). They proved the convergence of both algorithms under the condition $\|A\|<1$. As an illustration in a general context of descent methods for semi-algebraic problems, Attouch et al [1] showed that IHT algorithm (including an additional step size) for the regularized problem (1.4) actually converges for 
any matrix $A$.

Contributions and outline The bulk of this paper is to present and study a new nonsmooth nonconvex penalty for the regularized problem (1.4). Starting in $\S 2$ from the computation of the convex hull of $\mathrm{G}_{\ell_{0}}$ in the one dimensional case and in the case of orthogonal matrices (see $\S 3$ ), we propose a new relaxation of problem (1.4) by introducing a nonsmooth nonconvex but continuous penalty term that we call Continuous Exact $\ell_{0}$ (CEL0) penalty. This penalty can be viewed as a generalization of the penalty associated to the hard tresholding. In $\S 4$ we study the objective function of (1.4) where the $\ell_{0}$-norm is replaced by the CEL0 penalty for any matrix $A \in \mathbb{R}^{M \times N}$. Particularly, using the description of the minimizers of $\mathrm{G}_{\ell_{0}}$ given by Nikolova [32], we prove two main theoretical results concerning the relations between minimizers of the initial function $\mathrm{G}_{\ell_{0}}$ and its approximation using the CEL0 penalty. The global minimizers of this approximation contain the global minimizers of $\mathrm{G}_{\ell_{0}}$ and from each global minimizer of the CEL0 functional, a global minimizer of $\mathrm{G}_{\ell_{0}}$ can be easily computed. Similarly, from each local minimizer of the CEL0 functional, one can extract a local minimizer of $\mathrm{G}_{\ell_{0}}$. Moreover, this relaxed functional eliminates a significant number of strict local minimizers of $\mathrm{G}_{\ell_{0}}$ although this is not quantified theoretically. In other words, the extracted strict local minimizers of the proposed functional are less numerous than those of $\mathrm{G}_{\ell_{0}}$. Numerical experiments illustrate this fact. Hence, one can solve problem (1.4) by minimizing the sum of the quadratic data term and the CEL0 penalty. This can be addressed using numerous recent nonsmooth nonconvex algorithms converging to a critical point of the objective function. A small review of such algorithms will be outlined in $\S 5$. Based on this kind of algorithms and the properties of the CEL0 penalty, we propose in $\$ 5$ a macro algorithm by adding an outer loop to ensure the convergence to a point which is both a critical point of the relaxed functional and a (local) minimizer of $\mathrm{G}_{\ell_{0}}$.

Notations Let us first introduce some notations (we will use, in part, the same as in [32]):

- $\mathbb{I}_{N}=\{1, \cdots, N\}$,

- $a_{i} \in \mathbb{R}^{M}$, the $i$ th column of $A \in \mathbb{R}^{M \times N}$. We assume that $a_{i} \neq 0_{\mathbb{R}^{M}}, \forall i \in \mathbb{I}_{N}$,

- $\|\cdot\|=\|\cdot\|_{2}$ the $\ell_{2}$-norm. Otherwise we will precise the norm with a subscript,

- $e_{i} \in \mathbb{R}^{N}$, the unitary vector of the standard basis of $\mathbb{R}^{N}$,

- $\mathrm{x}^{(i)}=\left(\mathrm{x}_{1}, \cdots, \mathrm{x}_{i-1}, 0, \mathrm{x}_{i+1}, \cdots, \mathrm{x}_{N}\right) \in \mathbb{R}^{N}$,

- $A_{\omega}=\left(a_{\omega[1]}, \cdots, a_{\omega[\# \omega]}\right) \in R^{M \times \# \omega}$ for $\omega \subseteq \mathbb{I}_{N}$, the restriction of $A \in \mathbb{R}^{M \times N}$ to the columns indexed by the elements of $\omega \subseteq \mathbb{I}_{N}$,

- $\mathrm{x}_{\omega}=\left(\mathrm{x}_{\omega[1]}, \cdots, \mathrm{x}_{\omega[\# \omega]}\right) \in \mathbb{R}^{\# \omega}$ for $\omega \subseteq \mathbb{I}_{N}$, the restriction of $\mathrm{x} \in \mathbb{R}^{N}$ to the entries indexed by the elements of $\omega \subseteq \mathbb{I}_{N}$,

- $\sigma(\mathrm{x})=\left\{i \in \mathbb{I}_{N} ; \mathrm{x}_{i} \neq 0\right\} \subseteq \mathbb{I}_{N}$, the support of $\mathrm{x} \in \mathbb{R}^{N}$,

- $\sigma^{-}(\mathrm{x}) \subseteq \mathbb{I}_{N}$ and $\sigma^{+}(\mathrm{x}) \subseteq \mathbb{I}_{N}$ are two subsets of indexes which will be defined respectively by equations (4.5) and (4.6).

\section{The convex envelope in one dimension}

In this section we will focus on the one dimensional problem defined by

$$
\hat{u}=\underset{u \in \mathbb{R}}{\arg \min } g(u):=\frac{1}{2}(a u-d)^{2}+\lambda|u|_{0},
$$


where $a>0$ represents, in one dimension, the matrix $A \in \mathbb{R}^{M \times N}$ in (1.4) and $d \in \mathbb{R}$. Note that we can consider $a>0$ without loss of generality since $\mathrm{g}$ remains identical by multiplying both $a$ and $d$ by -1 . Here $|u|_{0}$ denotes the $\ell_{0}$-norm in one dimension,

$$
\forall u \in \mathbb{R},|u|_{0}= \begin{cases}0 & \text { if } u=0, \\ 1 & \text { if } u \neq 0 .\end{cases}
$$

It is well known that the convex envelope or convex hull of a function $f: \mathbb{R} \rightarrow \mathbb{R} \cup\{+\infty\}$ is also its biconjugate which is given by applying twice the Legendre-Fenchel transformation,

$$
f^{\star}\left(u^{\star}\right)=\sup _{u \in \mathbb{R}} u^{\star} u-f(u) .
$$

By applying the Legendre-Fenchel transformation to $g$ we obtain the conjugate $\mathrm{g}^{\star}$ (see Appendix A for details),

$$
\mathrm{g}^{\star}\left(u^{\star}\right)=\left\{\frac{1}{2 a^{2}}\left(u^{\star}+a d\right)^{2}-\lambda\right\} \mathbb{1}_{\left\{\left|u^{\star}+a d\right| \geq \sqrt{2 \lambda} a\right\}}-\frac{d^{2}}{2} .
$$

where $\mathbb{1}_{\{u \in E\}}$ denotes the indicator function defined by,

$$
\mathbb{1}_{\{u \in E\}}:= \begin{cases}1 & \text { if } u \in E, \\ 0 & \text { if } u \notin E .\end{cases}
$$

Then, we can determine the biconjugate $\mathrm{g}^{\star \star}$ which is the conjugate of $\mathrm{g}^{\star}$. As shown in Appendix A we have

$$
\mathrm{g}^{\star \star}(u)= \begin{cases}-a d u+|u| \sqrt{2 \lambda} a+\frac{d^{2}}{2} & \text { if }|u| \leq \frac{\sqrt{2 \lambda}}{a}, \\ \frac{1}{2}(a u-d)^{2}+\lambda & \text { if }|u| \geq \frac{\sqrt{2 \lambda}}{a} .\end{cases}
$$

Note that the computation of the 1D convex hull can also be found in [15]. This convex envelope is the largest lower-semi-continuous (l.s.c) convex function less than g. It is of major importance in global optimization. The first line of (2.6) can be rewritten as

$$
\frac{1}{2}(a u-d)^{2}-\frac{a^{2} u^{2}}{2}+|u| \sqrt{2 \lambda} a=\frac{1}{2}(a u-d)^{2}-\frac{a^{2}}{2}\left(|u|-\frac{\sqrt{2 \lambda}}{a}\right)^{2}+\lambda,
$$

and then,

$$
\mathrm{g}^{\star \star}(u)=\frac{1}{2}(a u-d)^{2}+\phi(a, \lambda ; u),
$$

where

$$
\phi(a, \lambda ; u)=\lambda-\frac{a^{2}}{2}\left(|u|-\frac{\sqrt{2 \lambda}}{a}\right)^{2} \mathbb{1}_{\left\{|u| \leq \frac{\sqrt{2 \lambda}}{a}\right\}},
$$

Finally, the convex envelope of $\mathrm{g}$ can be obtained by replacing the $\ell_{0}$ regularization term in (2.1) by the penalty given in (2.9). We can remark that the penalty term (2.9) is nonsmooth, nonconvex but continuous (see Figure 1 right) and when we combine it with the quadratic data term, the global objective function (2.8) is convex and gives the convex hull of g. Figure 1 presents two examples of the convex hull of $\mathrm{g}$ where the global minimum is respectively 0 and strictly positive. We also plot (Figure 1 right) the penalty (2.9) on the same graph as the $\ell_{0}$-norm. We can see that this penalty admits an horizontal asymptote which is a characteristic for unbiased solution (i.e. large coefficients are not shrinked) and is singular at the origin which is a necessary condition to provide sparse solutions according to Fan and Li [17]. 

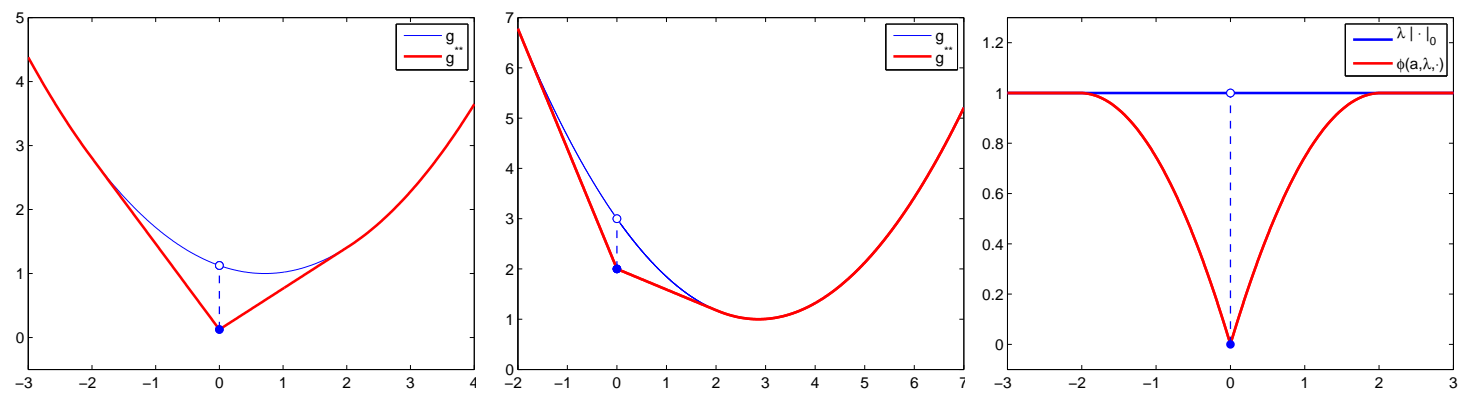

Figure 1: Plot of $\mathrm{g}$ (blue) and $\mathrm{g}^{\star \star}$ (red) for $a=0.7, \lambda=1$ and $d=0.5$ (left) or $d=2$ (center). Right: plot of $\lambda|\cdot|_{0}$ (blue) and $\phi(a, \lambda ; \cdot)$ for $a=0.7$ and $\lambda=1$.

\section{The convex hull when $A$ is orthogonal}

Extending the results of the previous section to the dimension $N \in \mathbb{N}$ is very tricky. Indeed, the conjugate of the function $\mathrm{G}_{\ell_{0}}: \mathbb{R}^{N} \rightarrow \mathbb{R}$ (eq. 1.4) is given by

$$
\mathrm{G}_{\ell_{0}}^{\star}\left(\mathrm{x}^{\star}\right)=\sup _{\mathrm{x} \in \mathbb{R}^{N}}\left\{\left\langle\mathrm{x}^{\star}, \mathrm{x}\right\rangle_{\mathbb{R}^{N}}-\frac{1}{2}\|A \mathrm{x}-d\|^{2}-\lambda\|\mathrm{x}\|_{0}\right\}
$$

which is also a combinatorial problem and probably as hard as the initial one given in (1.4). However, in the case where the matrix $A$ is orthogonal (i.e. $A^{T} A$ is diagonal), problem (3.1) can be solved analytically. Let $D \in \mathbb{R}^{N \times N}$ be a diagonal matrix whose diagonal entries are given by $d_{i}=\left\|a_{i}\right\| \forall i \in$ $\mathbb{I}_{N}$. Note that $D^{-1}$ is well defined since by hypothesis $\left\|a_{i}\right\| \neq 0 \forall i \in \mathbb{I}_{N}$. Let $\hat{d}=A D^{-2} A^{T} d$ and $\tilde{z}=D^{-1} A^{T} d$, then the quadratic term of (3.1) can be written as

$$
\frac{1}{2}\|A \mathrm{x}-d\|^{2}=\frac{1}{2}\|d-\hat{d}\|^{2}+\frac{1}{2}\|D \mathrm{x}-\tilde{z}\|^{2} .
$$

Combining (3.1) and (3.2) we obtain,

$$
\begin{aligned}
\mathrm{G}_{\ell_{0}}^{\star}\left(\mathrm{x}^{\star}\right) & =\sup _{\mathrm{x} \in \mathbb{R}^{N}}\left\langle\mathrm{x}^{\star}, \mathrm{x}\right\rangle_{\mathbb{R}^{N}}-\frac{1}{2}\|d-\hat{d}\|^{2}-\frac{1}{2}\|D \mathrm{x}-\tilde{z}\|^{2}-\lambda\|\mathrm{x}\|_{0} \\
& =-\frac{1}{2}\|d-\hat{d}\|^{2}+\sup _{\mathrm{x} \in \mathbb{R}^{N}} \sum_{i \in \mathbb{I}_{N}} \mathrm{x}_{i}^{\star} \mathrm{x}_{i}-\frac{1}{2}\left(\left\|a_{i}\right\| \mathrm{x}_{i}-\tilde{z}_{i}\right)^{2}-\lambda\left|\mathrm{x}_{i}\right|_{0} \\
& =-\frac{1}{2}\|d-\hat{d}\|^{2}+\sum_{i \in \mathbb{I}_{N}} \sup _{\mathrm{x}_{i} \in \mathbb{R}} \mathrm{x}_{i}^{\star} \mathrm{x}_{i}-\frac{1}{2}\left(\left\|a_{i}\right\| \mathrm{x}_{i}-\tilde{z}_{i}\right)^{2}-\lambda\left|\mathrm{x}_{i}\right|_{0}
\end{aligned}
$$

Finally, when $A$ is orthogonal, solving (3.1) is equivalent to solve $N$ independent one dimensional problems. Using the one dimensional conjugate $\mathrm{g}^{\star}$ given by $(2.4)$ it comes that

$$
\mathrm{G}_{\ell_{0}}^{\star}\left(\mathrm{x}^{\star}\right)=-\frac{1}{2}\|d-\hat{d}\|^{2}+\sum_{i \in \mathbb{I}_{N}}\left\{\frac{1}{2\left\|a_{i}\right\|^{2}}\left(\mathrm{x}_{i}^{\star}+\left\|a_{i}\right\| \tilde{z}_{i}\right)^{2}-\lambda\right\} \mathbb{1}_{\left\{\left|\mathrm{x}_{i}^{\star}+\left\|a_{i}\right\| \tilde{z}_{i}\right| \geq \sqrt{2 \lambda}\left\|a_{i}\right\|\right\}}-\frac{\tilde{z}_{i}^{2}}{2}
$$

which is additively separable and allows to use the expression of the one dimensional convex hull $\mathrm{g}^{\star \star}$ 
given by (2.8) to get,

$$
\begin{aligned}
\mathrm{G}_{\ell_{0}}^{\star \star}(\mathrm{x}) & =\frac{1}{2}\|d-\hat{d}\|^{2}+\sum_{i \in \mathbb{I}_{N}} \frac{1}{2}\left(\left\|a_{i}\right\| \mathrm{x}_{i}-\tilde{z}_{i}\right)^{2}+\phi\left(\left\|a_{i}\right\|, \lambda ; \mathrm{x}_{i}\right), \\
& =\frac{1}{2}\|d-\hat{d}\|^{2}+\frac{1}{2}\|D \mathrm{x}-\tilde{z}\|^{2}+\sum_{i \in \mathbb{I}_{N}} \phi\left(\left\|a_{i}\right\|, \lambda ; \mathrm{x}_{i}\right), \\
& =\frac{1}{(3.2)}\|A \mathrm{x}-d\|^{2}+\sum_{i \in \mathbb{I}_{N}} \phi\left(\left\|a_{i}\right\|, \lambda ; \mathrm{x}_{i}\right) .
\end{aligned}
$$

Note that, as in the one dimensional case, the convex hull of $\mathrm{G}_{\ell_{0}}$ for an orthogonal matrix $A$ is obtained by replacing the non continuous $\ell_{0}$-norm by a continuous penalty defined as in (3.5) with $\phi$ given by (2.9).

\section{The CEL0 penalty}

Extending the study of the previous section to the general case with an arbitrary matrix $A$ seems to be as hard as the initial problem (1.4). However, the relaxed penalty obtained in the case of orthogonal matrices remains interesting even when $A$ is not orthogonal. The goal of this section is then to study the relaxed functional,

$$
\mathrm{G}_{\mathrm{CEL}}(\mathrm{x}):=\frac{1}{2}\|A \mathrm{x}-d\|^{2}+\Phi_{\mathrm{cEL}}(\mathrm{x}),
$$

for an arbitrary $A \in \mathbb{R}^{M \times N}$ and where $\Phi_{\mathrm{CEL}}(\cdot)$ is the Continuous Exact $\ell_{0}(\mathrm{CEL} 0)$ penalty defined by,

$$
\Phi_{\mathrm{cEL} 0}(\mathrm{x}):=\sum_{i \in \mathbb{I}_{N}} \phi\left(\left\|a_{i}\right\|, \lambda, \mathrm{x}_{i}\right)=\sum_{i \in \mathbb{I}_{N}} \lambda-\frac{\left\|a_{i}\right\|^{2}}{2}\left(\left|\mathrm{x}_{i}\right|-\frac{\sqrt{2 \lambda}}{\left\|a_{i}\right\|}\right)^{2} \mathbb{1}_{\left\{\left|\mathrm{x}_{i}\right| \leq \sqrt{2 \lambda}\right\}},
$$

for $\lambda>0$. The name CEL0 of this penalty comes from the two main results of the objective function $\mathrm{G}_{\text {СЕLo }}$ which are presented in the following. The first one, given in Theorem 4.5, states that global minimizers of $\mathrm{G}_{\text {CEL }}$ contain global minimizers of $\mathrm{G}_{\ell_{0}}$ and that from each global minimizer of $\mathrm{G}_{\text {CEL }}$, a global minimizer of $\mathrm{G}_{\ell_{0}}$ can be easily obtained. The second one, given in Theorem 4.8, partially extends this result to local minimizers and numerical illustrations show that, in general, a large number of strict local minimizers of $\mathrm{G}_{\ell_{0}}$ are not critical points of $\mathrm{G}_{\text {CELO }}$. Therefore $\mathrm{G}_{\text {CЕLо }}$ admits "less" strict local minimizers than $\mathrm{G}_{\ell_{0}}$. Although $\mathrm{G}_{\text {CEL }}$ is nonconvex (for a nonorthogonal matrix $A$ ), it is continuous and recent nonsmooth nonconvex optimization algorithms can be used to minimize it and thus minimize $\mathrm{G}_{\ell_{0}}$. This point will be developed in $\S 5$.

Remark 4.1. One can easily see that $\mathrm{G}_{\mathrm{CEL}}$ underestimate $\mathrm{G}_{\ell_{0}}$. For $\lambda>0$ we have $0 \leq \phi\left(\left\|a_{i}\right\|, \lambda ; u\right) \leq$ $1 \forall u \in \mathbb{R}, \forall i \in \mathbb{I}_{N}$. Then, since $\phi\left(\left\|a_{i}\right\|, \lambda ; 0\right)=0$ it comes that $\phi\left(\left\|a_{i}\right\|, \lambda ; u\right) \leq|u|_{0}, \forall u \in \mathbb{R}, \forall i \in \mathbb{I}_{N}$, which proves the above affirmation.

We begin by a characterization of the critical points of $\mathrm{G}_{\mathrm{CEL} \perp}$. Let us start with the expression of

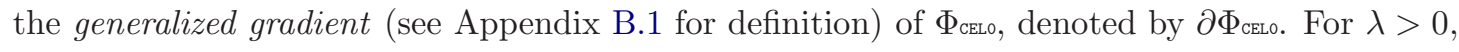

$$
\partial \Phi_{\mathrm{CEL} O}(\mathrm{x})=\prod_{i \in \mathbb{I}_{N}} \sqrt{2 \lambda}\left\|a_{i}\right\|[-1,1] \mathbb{1}_{\left\{\mathrm{x}_{i}=0\right\}}+\left\|a_{i}\right\|\left\{\operatorname{sign}\left(\mathrm{x}_{i}\right) \sqrt{2 \lambda}-\left\|a_{i}\right\| \mathrm{x}_{i}\right\} \mathbb{1}_{\left\{0<\left|\mathrm{x}_{i}\right|<\frac{\sqrt{2 \lambda}}{\left\|a_{i}\right\|}\right\}} .
$$


This expression comes directly from the generalized derivative of the one dimensional penalty $\phi$ (see Appendix B.1 for details). The following lemma gives a characterization of the critical points of $G_{\text {CELO }}$. Such points $\mathrm{x} \in \mathbb{R}^{N}$ verify $0_{\mathbb{R}^{N}} \in \partial \mathrm{G}_{\mathrm{CEL} \mathrm{O}}(\mathrm{x})$ (necessary condition, see Proposition B.1).

Lemma 4.1 (Critical points of $\mathrm{G}_{\mathrm{CELO}}$ ). Let $\mathrm{G}_{\mathrm{CELO}}$ be defined by (4.1) for $d \in \mathbb{R}^{M}$ and $\lambda>0$. Let $s_{i}=\operatorname{sign}\left(\left\langle a_{i}, A \hat{\mathrm{x}}^{(i)}-d\right\rangle\right)$ and $\mathrm{x}^{(i)}=\left(\mathrm{x}_{1}, \cdots, \mathrm{x}_{i-1}, 0, \mathrm{x}_{i+1}, \cdots, \mathrm{x}_{N}\right)$. Then $\hat{\mathrm{x}} \in \mathbb{R}^{N}$ is a critical point of $\mathrm{G}_{\mathrm{CELO}}$ (i.e. $\left.0_{\mathbb{R}^{N}} \in \partial \mathrm{G}_{\mathrm{CELO}}(\hat{\mathrm{x}})\right)$ if and only if

$$
\forall i \in \mathbb{I}_{N} \begin{cases}\hat{\mathrm{x}}_{i}=0 & \text { iff }\left|\left\langle a_{i}, A \hat{\mathrm{x}}^{(i)}-d\right\rangle\right| \leq \sqrt{2 \lambda}\left\|a_{i}\right\|, \\ \hat{\mathrm{x}}_{i}=-s_{i} t, t \in\left[0, \frac{\sqrt{2 \lambda}}{\left\|a_{i}\right\|}\right] & \text { iff }\left|\left\langle a_{i}, A \hat{\mathrm{x}}^{(i)}-d\right\rangle\right|=\sqrt{2 \lambda}\left\|a_{i}\right\|, \\ \hat{\mathrm{x}}_{i}=-\frac{\left\langle a_{i}, A \hat{\mathrm{x}}^{(i)}-d\right\rangle}{\left\|a_{i}\right\|^{2}} & \text { iff }\left|\left\langle a_{i}, A \hat{\mathrm{x}}^{(i)}-d\right\rangle\right| \geq \sqrt{2 \lambda}\left\|a_{i}\right\| .\end{cases}
$$

Proof. The proof is outlined in Appendix B.2.

The characterization provided by Lemma 4.1 will be useful to provide bounds on the nonzero entries of (local) minimizers. We introduce two subsets used in the following,

$$
\forall \mathrm{x} \in \mathbb{R}^{N}, \sigma^{-}(\mathrm{x}):=\left\{i \in \mathbb{I}_{N}: 0<\left|\mathrm{x}_{i}\right|<\frac{\sqrt{2 \lambda}}{\left\|a_{i}\right\|}\right\} \subseteq \sigma(\mathrm{x}),
$$

and for a critical point $\hat{\mathrm{x}} \in \mathbb{R}^{N}$ of $\mathrm{G}_{\mathrm{CELO}}$,

$$
\begin{aligned}
\sigma^{+}(\hat{\mathrm{x}}) & :=\left\{i:\left|\left\langle a_{i}, A \hat{\mathrm{x}}^{(i)}-d\right\rangle\right|=\sqrt{2 \lambda}\left\|a_{i}\right\|\right\} \\
& =\left\{i: \hat{\mathrm{x}}_{i}=0 \text { and }\left|\left\langle a_{i}, A \hat{\mathrm{x}}^{(i)}-d\right\rangle\right|=\sqrt{2 \lambda}\left\|a_{i}\right\|\right\} \cup\left\{i: 0<\left|\mathrm{x}_{i}\right| \leq \frac{\sqrt{2 \lambda}}{\left\|a_{i}\right\|}\right\} .
\end{aligned}
$$

\subsection{On the minimizers of $\mathrm{G}_{\text {CELO }}$}

Let us recall two results given in [32]. The first one provides a lower bound on the nonzero coefficients of the global minimizers of $\mathrm{G}_{\ell_{0}}$ while the second one characterizes the (local) minimizers of $\mathrm{G}_{\ell_{0}}$.

Proposition 4.2 (M. Nikolova [32]). For $d \in \mathbb{R}^{M}$ and $\lambda>0$, let $\mathrm{G}_{\ell_{0}}$ have a global minimum at $\hat{\mathrm{x}} \in \mathbb{R}^{N}$. Then

$$
i \in \sigma(\hat{\mathrm{x}}) \Longrightarrow\left|\hat{\mathrm{x}}_{i}\right| \geq \frac{\sqrt{2 \lambda}}{\left\|a_{i}\right\|},
$$

Proof. The proof is given in [32, Appendix 8.2]. This result is also known from [31, Proposition 3.4] in a more general setting.

Corollary 4.3 (M. Nikolova [32]). For $d \in \mathbb{R}^{M}$ and $\lambda>0$, let $\hat{\mathrm{x}} \in \mathbb{R}^{N}$ be a (local) minimizer of $\mathrm{G}_{\ell_{0}}$. Set $\hat{\sigma}=\sigma(\hat{\mathrm{x}})$. Then

$$
\hat{\mathrm{x}}_{\hat{\sigma}} \text { solves }\left(A_{\hat{\sigma}}\right)^{T} A_{\hat{\sigma}} \hat{\mathrm{x}}_{\hat{\sigma}}=\left(A_{\hat{\sigma}}\right)^{T} d .
$$

Conversely, if $\hat{\mathrm{x}} \in \mathbb{R}^{N}$ verifies (4.8) for $\hat{\sigma}=\sigma(\hat{\mathrm{x}})$, then $\hat{\mathrm{x}}$ is a (local) minimizer of $\mathrm{G}_{\ell_{0}}$.

Proof. The proof follows directly from [32, Lemma 2.4] for (4.8) and [32, Proposition 2.3] for the reciprocal. 
For the function $\mathrm{G}_{\text {CEL }}$ we have a result similar to the one given in Proposition 4.2 which is provided by the following lemma.

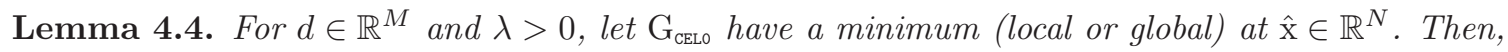

$$
\forall i \in \sigma^{+}(\hat{\mathrm{x}}), \forall t \in\left[0, \frac{\sqrt{2 \lambda}}{\left\|a_{i}\right\|}\right],\left(\hat{\mathrm{x}}^{(i)}-s_{i} e_{i} t\right) \text { is a minimizer of } \mathrm{G}_{\mathrm{CEL} O} \text {. }
$$

where $s_{i}=\operatorname{sign}\left(\left\langle a_{i}, A \hat{\mathrm{x}}^{(i)}-d\right\rangle\right)$. Furthermore, $t \mapsto \mathrm{G}_{\mathrm{CEL} 0}\left(\hat{\mathrm{x}}^{(i)}-s_{i} e_{i} t\right)$ is constant on $\left[0, \frac{\sqrt{2 \lambda}}{\left\|a_{i}\right\|}\right]$.

Proof. The proof comes directly from Lemma 4.1 which gives a characterization of the critical points of $\mathrm{G}_{\text {CELO }}$. Indeed, since $\hat{\mathrm{x}}$ is a minimizer (local or global) of $\mathrm{G}_{\mathrm{CELO}}$ it has to verify the conditions given in (4.4) from which we get

$$
\forall i \in \sigma^{+}(\hat{\mathrm{x}}), \exists \hat{t} \in\left[0, \frac{\sqrt{2 \lambda}}{\left\|a_{i}\right\|}\right] \quad \text { s.t. } \hat{\mathrm{x}}_{i}=\operatorname{sign}\left(\hat{\mathrm{x}}_{i}\right) \hat{t}=-s_{i} \hat{t},
$$

since, from Appendix B.2, $s_{i}=\operatorname{sign}\left(\left\langle a_{i}, A \hat{\mathrm{x}}^{(i)}-d\right\rangle\right)=-\operatorname{sign}\left(\hat{\mathrm{x}}_{i}\right)$. Let $i \in \sigma^{+}(\hat{\mathrm{x}})$ and $f$ be the restriction of $\mathrm{G}_{\mathrm{CELO}}$ defined by

$$
f(t)=\mathrm{G}_{\mathrm{CEL} 0}\left(\hat{\mathrm{x}}^{(i)}-s_{i} e_{i} t\right), \forall t \in\left[0, \frac{\sqrt{2 \lambda}}{\left\|a_{i}\right\|}\right] .
$$

We can rewrite $f$ as follows, $\forall t \in\left[0, \frac{\sqrt{2 \lambda}}{\left\|a_{i}\right\|}\right]$

$$
\begin{aligned}
f(t) & =\frac{1}{2}\left\|A \hat{\mathrm{x}}^{(i)}-s_{i} a_{i} t-d\right\|^{2}+\sum_{j \in \mathbb{I}_{N}, j \neq i} \phi\left(\left\|a_{j}\right\|, \lambda ; \hat{\mathrm{x}}_{j}\right)+\phi\left(\left\|a_{i}\right\|, \lambda ;-s_{i} t\right) \\
& =C-s_{i} t\left\langle a_{i}, A \hat{\mathrm{x}}^{(i)}-d\right\rangle+\frac{\left\|a_{i}\right\|^{2}}{2} t^{2}+\lambda-\frac{\left\|a_{i}\right\|^{2}}{2}\left(\left|s_{i} t\right|-\frac{\sqrt{2 \lambda}}{\left\|a_{i}\right\|}\right)^{2} \\
& =C-s_{i} t\left\langle a_{i}, A \hat{\mathrm{x}}^{(i)}-d\right\rangle+t \sqrt{2 \lambda}\left\|a_{i}\right\| \\
& =C+t\left(\sqrt{2 \lambda}\left\|a_{i}\right\|-\left|\left\langle a_{i}, A \hat{\mathrm{x}}^{(i)}-d\right\rangle\right|\right)=C=f(\hat{t}),
\end{aligned}
$$

since by definition (eq. 4.6) $i \in \sigma^{+}(\hat{\mathrm{x}}) \Rightarrow\left|\left\langle a_{i}, A \hat{\mathrm{x}}^{(i)}-d\right\rangle\right|=\sqrt{2 \lambda}\left\|a_{i}\right\|$ and $C=\frac{1}{2}\left\|A \hat{\mathrm{x}}^{(i)}-d\right\|^{2}+$ $\sum_{j \in \mathbb{I}_{N}, j \neq i} \phi\left(\left\|a_{j}\right\|, \lambda ; \hat{\mathrm{x}}_{j}\right)$ is a constant independent of $t$. Using the fact that $\hat{\mathrm{x}}$ is a minimizer (local or global) of $\mathrm{G}_{\mathrm{CEL}},(4.12)$ completes the proof.

Remark 4.2. In the light of Lemma 4.4, one can interpret $\sigma^{+}(\hat{\mathrm{x}})$ (for $\hat{\mathrm{x}}$ critical point of $\left.\mathrm{G}_{\mathrm{CEL}}\right)$ as the set of indexes such that $t \mapsto \mathrm{G}_{\mathrm{CELO}}\left(\hat{\mathrm{x}}^{(i)}-s_{i} e_{i} t\right)$ is constant on $\left[0, \frac{\sqrt{2 \lambda}}{\left\|a_{i}\right\|}\right]$.

This lemma ensures that all strict minimizers of $\mathrm{G}_{\mathrm{CELO}}$ - i.e. $\hat{\mathrm{x}} \in \mathbb{R}^{N}$ such that there exists a neighborhood $\mathcal{V} \subset \mathbb{R}^{N}$ containing $\hat{\mathrm{x}}$ for which $\forall \mathrm{y} \in \mathcal{V} \backslash\{\hat{\mathrm{x}}\}, \mathrm{G}_{\text {CELO }}(\hat{\mathrm{x}})<\mathrm{G}_{\text {CELO }}(\mathrm{y})-\operatorname{verify} \sigma^{+}(\hat{\mathrm{x}})=\emptyset$. Indeed, suppose that this claim is not verified by a strict minimizer $\hat{\mathrm{x}}$ of $\mathrm{G}_{\mathrm{CEL} O}$, then Lemma 4.4 states that $\forall i \in \sigma^{+}(\hat{\mathrm{x}}), \forall t \in\left[0, \frac{\sqrt{2 \lambda}}{\left\|a_{i}\right\|}\right], \hat{\mathrm{x}}^{(i)}-s_{i} e_{i} t$ is also a minimizer of $\mathrm{G}_{\text {CELo }}$ and contradicts the fact that $\hat{\mathrm{x}}$ is strict.

Moreover, for a nonstrict minimizer $\hat{\mathrm{x}} \in \mathbb{R}^{N}$, fixing all its nonzero components indexed by $\sigma^{-}(\hat{\mathrm{x}})$ to zero will result, from Lemma 4.4 , in another minimizer $\hat{\mathrm{x}}^{0}$ defined by,

$$
\forall i \in \mathbb{I}_{N}, \hat{\mathrm{x}}_{i}^{0}:=\left\{\begin{array}{ll}
\hat{\mathrm{x}}_{i} & \text { if } i \notin \sigma^{-}(\hat{\mathrm{x}}) \\
0 & \text { if } i \in \sigma^{-}(\hat{\mathrm{x}})
\end{array}=\hat{\mathrm{x}}_{i} \mathbb{1}_{\left\{\left|\hat{\mathrm{x}}_{i}\right| \geq \frac{\sqrt{2 \lambda}}{\left\|a_{i}\right\|}\right\}},\right.
$$


and verifying,

$$
\mathrm{G}_{\mathrm{CELO}}\left(\hat{\mathrm{x}}^{0}\right)=\mathrm{G}_{\mathrm{CELO}}(\hat{\mathrm{x}}) .
$$

It is obvious that such an $\hat{\mathrm{x}}^{0}$ verifies $\sigma^{-}\left(\hat{\mathrm{x}}^{0}\right)=\emptyset$ but is not strict by definition. Based on this lemma, the following theorem gives a relation between the global minimizers of $\mathrm{G}_{\ell_{0}}$ and $\mathrm{G}_{\mathrm{CEL}}$.

Theorem 4.5 (Link between global minimizers of $\mathrm{G}_{\ell_{0}}$ and $\mathrm{G}_{\mathrm{CELO}}$ ). Let $d \in \mathbb{R}^{M}$ and $\lambda>0$,

(i) the set of global minimizers of $\mathrm{G}_{\ell_{0}}$ is included in the set of global minimizers of $\mathrm{G}_{\text {CЕLo }}$,

$$
\underset{\mathrm{x} \in \mathbb{R}^{N}}{\arg \min } \mathrm{G}_{\ell_{0}}(\mathrm{x}) \subseteq \underset{\mathrm{x} \in \mathbb{R}^{N}}{\arg \min } \mathrm{G}_{\mathrm{CEED}}(\mathrm{x}),
$$

(ii) conversely if $\hat{\mathrm{x}} \in \mathbb{R}^{N}$ is a global minimizer of $\mathrm{G}_{\mathrm{CEL}}$, then $\hat{\mathrm{x}}^{0}$ (defined by (4.13)) is a global minimizer of $\mathrm{G}_{\ell_{0}}$ and

$$
\mathrm{G}_{\mathrm{CELO}}(\hat{\mathrm{x}})=\mathrm{G}_{\mathrm{CELO}}\left(\hat{\mathrm{x}}^{0}\right)=\mathrm{G}_{\ell_{0}}\left(\hat{\mathrm{x}}^{0}\right) \text {. }
$$

Proof. The proof is detailed in Appendix C.

Remark 4.3. Since $\hat{\mathrm{x}}^{0}$ is a global minimizer of $\mathrm{G}_{\ell_{0}}$, it is strict [32, Theorem 4.4 (ii)] and we can conclude from [32, Theorem 3.2] that $A_{\sigma\left(\hat{\mathrm{x}}^{0}\right)}$ has a full column rank. Hence, denoting $\hat{\sigma}^{0}=\sigma\left(\hat{\mathrm{x}}^{0}\right)$, we have

$$
\hat{\mathrm{x}}_{\hat{\sigma}^{0}}^{0}=\left(\left(A_{\hat{\sigma}^{0}}\right)^{T} A_{\hat{\sigma}^{0}}\right)^{-1}\left(A_{\hat{\sigma}^{0}}\right)^{T} d \text { and } \hat{\mathrm{x}}_{\mathbb{I}_{N} \backslash \hat{\sigma}^{0}}^{0}=0 .
$$

Proposition 4.6 (Existence of global minimizers for $\mathrm{G}_{\text {СЁо }}$ ). The set of global minimizers of $\mathrm{G}_{\text {СЕLо }}$ is nonempty.

Proof. [32, Theorem 4.4 (i)] states that the set of global minimizers of $\mathrm{G}_{\ell_{0}}$ is nonempty. Then the result for $\mathrm{G}_{\text {CELO }}$ is straightforward from Theorem 4.5 (i).

The following lemma characterizes some critical points of $\mathrm{G}_{\text {CELO }}$ as (local) minimizers of $\mathrm{G}_{\ell_{0}}$.

Lemma 4.7 (Link between critical points of $\mathrm{G}_{\text {CЕLo }}$ and minimizers of $\mathrm{G}_{\ell_{0}}$ ). Let $\hat{\mathrm{x}} \in \mathbb{R}^{N}$ be a critical point of $\mathrm{G}_{\mathrm{CELO}}$ verifying $\sigma^{-}(\hat{\mathrm{x}})=\emptyset$. Then it is a (local) minimizer of $\mathrm{G}_{\ell_{0}}$ and $\mathrm{G}_{\mathrm{CEL}}(\hat{\mathrm{x}})=\mathrm{G}_{\ell_{0}}(\hat{\mathrm{x}})$.

Proof. From Lemma 4.1 we get that $\hat{\mathrm{x}}$, which is a critical point of $\mathrm{G}_{\mathrm{CELO}}$ such that $\sigma^{-}(\hat{\mathrm{x}})=\emptyset$ (i.e. $\left.\forall i \in \sigma(\hat{\mathrm{x}}),\left|\hat{\mathrm{x}}_{i}\right| \geq \frac{\sqrt{2 \lambda}}{\left\|a_{i}\right\|}\right)$, verifies

$$
\forall i \in \sigma(\hat{\mathrm{x}}), \hat{\mathrm{x}}_{i}=-\frac{\left\langle a_{i}, A \hat{\mathrm{x}}^{(i)}-d\right\rangle}{\left\|a_{i}\right\|^{2}} \Longleftrightarrow\left\langle a_{i}, A \hat{\mathrm{x}}-d\right\rangle=0
$$

Let $\hat{\sigma}=\sigma(\hat{\mathrm{x}})$, then we get

$$
\begin{aligned}
\left(A_{\hat{\sigma}}\right)^{T}(A \hat{\mathrm{x}}-d)=0 & \Longleftrightarrow\left(A_{\hat{\sigma}}\right)^{T}(A_{\hat{\sigma}} \hat{\mathrm{x}}_{\hat{\sigma}}+A_{\hat{\sigma}^{c}} \underbrace{\hat{\mathrm{x}}_{\hat{\sigma}^{c}}}_{=0}-d)=0, \\
& \Longleftrightarrow\left(A_{\hat{\sigma}}\right)^{T} A_{\hat{\sigma}} \hat{\mathrm{x}}_{\hat{\sigma}}=\left(A_{\hat{\sigma}}\right)^{T} d
\end{aligned}
$$

Then from Corollary 4.3 it comes that $\hat{\mathrm{x}}$ is a (local) minimizer for $\mathrm{G}_{\ell_{0}}$. The fact that $\mathrm{G}_{\text {сЕЕо }}(\hat{\mathrm{x}})=$ $\mathrm{G}_{\ell_{0}}(\hat{\mathrm{x}})$ follows from the same arguments as in the proof of Theorem 4.5. This completes the proof.

From this lemma we can derive a result similar to Theorem 4.5 for the local minimizers which are not global. 
Theorem 4.8 (Link between local minimizers of $\mathrm{G}_{\ell_{0}}$ and $\mathrm{G}_{\mathrm{CEL}}$ ). Let $d \in \mathbb{R}^{M}, \lambda>0$, and $\mathrm{G}_{\text {CELo }}$ have a local minimum (not global) at $\hat{\mathrm{x}} \in \mathbb{R}^{N}$. Then $\hat{\mathrm{x}}^{0}$ (defined as in (4.13)) is a local minimizer (not global) of $\mathrm{G}_{\ell_{0}}$ and (4.16) is verified.

Proof. From Lemma 4.4 we get that since $\hat{\mathrm{x}}$ is a local minimizer of $\mathrm{G}_{\text {CEL }}, \hat{\mathrm{x}}^{0}$ is also a local minimizer of $\mathrm{G}_{\text {CELO }}$. Thus, $\hat{\mathrm{x}}^{0}$ is a critical point of $\mathrm{G}_{\text {CELO }}$ such that $\sigma^{-}\left(\hat{\mathrm{x}}^{0}\right)=\emptyset$ and, from Lemma 4.7 , it is a (local) minimizer of $\mathrm{G}_{\ell_{0}}$ and (4.16) is verified. Moreover, according to Theorem 4.5 (i), it can not be global for $\mathrm{G}_{\ell_{0}}$ since it is not global for $\mathrm{G}_{\mathrm{CEL} \mathrm{O}}$. This completes the proof.

Remark 4.4. Although, for a global minimizer $\hat{\mathrm{x}}$ of $\mathrm{G}_{\mathrm{CEL} 0}, \hat{\mathrm{x}}^{0}$ is strict for $\mathrm{G}_{\ell_{0}}$ (see Remark 4.3), this is not always true for a local minimizer (not global) of $\mathrm{G}_{\mathrm{CEL} O}$. Indeed, let $\hat{\mathrm{x}}$ be a local minimizer (not global) of $\mathrm{G}_{\text {CELO }}$ such that $\operatorname{rank}\left(A_{\hat{\sigma}^{0}}\right) \leq \# \hat{\sigma}^{0}-1$ where $\hat{\sigma}^{0}=\sigma\left(\hat{\mathrm{x}}^{0}\right)$ and $\hat{\mathrm{x}}^{0}$ defined by (4.13) (we assume $\left.\hat{\sigma}^{0} \neq \emptyset\right)$. Then, from Theorem 4.8, $\hat{\mathrm{x}}^{0}$ is a local minimizer of $\mathrm{G}_{\ell_{0}}$ and [32, Theorem 3.2] ensures that $\hat{\mathrm{x}}^{0}$ is nonstrict for $\mathrm{G}_{\ell_{0}}$. Moreover let

$$
\left.\left.V:=\prod_{\substack{i \in \hat{\sigma}^{0} \\ \hat{\mathrm{x}}_{i}^{0} \leq-\frac{\sqrt{2 \lambda}}{\left\|a_{i}\right\|}}}\right]-\infty,\left|\hat{\mathrm{x}}_{i}^{0}\right|-\frac{\sqrt{2 \lambda}}{\left\|a_{i}\right\|}\right] \times \prod_{\substack{i \in \hat{\sigma}^{0} \\ \hat{\mathrm{x}}_{i}^{0} \geq \frac{\sqrt{2 \lambda}}{\left\|a_{i}\right\|}}}\left[\frac{\sqrt{2 \lambda}}{\left\|a_{i}\right\|}-\left|\hat{\mathrm{x}}_{i}^{0}\right|,+\infty\left[\subset \mathbb{R}^{\# \hat{\sigma}^{0}},\right.\right.
$$

then for any $v \in \mathbb{R}^{N}$ such that $v_{\hat{\sigma}^{0}} \in \operatorname{Ker}\left(A_{\hat{\sigma}^{0}}\right) \cap V$ and $v_{\mathbb{I}_{N} \backslash \hat{\sigma}^{0}}=0$ we have,

$$
\forall i \in \hat{\sigma}^{0},\left|\hat{\mathrm{x}}_{i}^{0}+v_{i}\right| \geq \frac{\sqrt{2 \lambda}}{\left\|a_{i}\right\|},
$$

and it is straightforward that $\sigma^{-}\left(\hat{\mathrm{x}}^{0}+v\right)=\emptyset$. Furthermore, one can see that

$$
\left(A_{\hat{\sigma}^{0}}\right)^{T}\left(A_{\hat{\sigma}^{0}}\left(\hat{\mathrm{x}}_{\hat{\sigma}^{0}}^{0}+v_{\hat{\sigma}^{0}}\right)-d\right)=\left(A_{\hat{\sigma}^{0}}\right)^{T}\left(A_{\hat{\sigma}^{0}} \hat{\mathrm{x}}_{\hat{\sigma}^{0}}^{0}-d\right)=0 .
$$

From (4.19), (4.20) and Lemma 4.1, we get that $\hat{\mathrm{x}}^{0}+v$ is a critical point of $\mathrm{G}_{\text {CELo }}$ which, from Lemma 4.7, is a local minimizer of $\mathrm{G}_{\ell_{0}}$.

Hence, if $\operatorname{rank}\left(A_{\hat{\sigma}^{0}}\right) \leq \# \hat{\sigma}^{0}-1, \hat{\mathrm{x}}^{0}$ is nonstrict for $\mathrm{G}_{\ell_{0}}$ on the polyhedron

$$
K:=\left\{\hat{\mathrm{x}}^{0}+v, v \in \mathbb{R}^{N}: v_{\hat{\sigma}^{0}} \in \operatorname{Ker}\left(A_{\hat{\sigma}^{0}}\right) \cap V \text { and } v_{\mathbb{I}_{N} \backslash \hat{\sigma}^{0}}=0\right\},
$$

of dimension,

$$
\begin{aligned}
\operatorname{dim} K & =\operatorname{dim}\left(\operatorname{Ker}\left(A_{\hat{\sigma}^{0}}\right) \cap V\right) \\
& =\operatorname{dim} \operatorname{Ker}\left(A_{\hat{\sigma}^{0}}\right)+\operatorname{dim} V-\operatorname{dim} \mathbb{R}^{\# \hat{\sigma}^{0}} \\
& =\operatorname{dim} \operatorname{Ker}\left(A_{\hat{\sigma}^{0}}\right) \\
& =\# \hat{\sigma}^{0}-\operatorname{rank}\left(A_{\hat{\sigma}^{0}}\right) \geq 1
\end{aligned}
$$

from the rank nullity theorem and using the fact that

$$
\operatorname{dim}\left(W_{1}+W_{2}\right)=\operatorname{dim} W_{1}+\operatorname{dim} W_{2}-\operatorname{dim}\left(W_{1} \cap W_{2}\right),
$$

for two subspaces $W_{1}$ and $W_{2}$ of a finite dimensional vector space $U$.

Remark 4.5. For a (local) minimizer $\hat{\mathrm{x}}$ of $\mathrm{G}_{\mathrm{CEL} O}$, one can set $\forall i \in \sigma^{+}(\hat{\mathrm{x}})$ either $\hat{\mathrm{x}}_{i}=0$ or $\hat{\mathrm{x}}_{i}=-s_{i} \frac{\sqrt{2 \lambda}}{\left\|a_{i}\right\|}$ to obtain a (local) minimizer $\mathrm{x}^{\star}$ of both $\mathrm{G}_{\text {CELO }}$ and $\mathrm{G}_{\ell_{0}}$ (Lemmas 4.4 and 4.7). There exists $2^{\# \sigma^{+}(\hat{\mathrm{x}})}$ of such minimizers for which Theorems 4.5 and 4.8 hold also. Among them, $\hat{\mathrm{x}}^{0}$ is the sparsest minimizer of $\mathrm{G}_{\ell_{0}}$ which can be obtained from $\hat{\mathrm{x}}$ by changing only the entries belonging to $\sigma^{-}(\hat{\mathrm{x}})$. Note that we can obtain a sparser minimizer than $\hat{\mathrm{x}}^{0}$ by thresholding all the entries indexed by $\sigma^{+}(\hat{\mathrm{x}})$. 
In other words, Theorem 4.8 states that the second point of Theorem 4.5 is also true for local minimizers of $\mathrm{G}_{\text {CELO }}$. However, the reciprocal is not verified for all the local minimizers of $\mathrm{G}_{\ell_{0}}$. Indeed, let us consider $N=1$ (i.e. problem 2.1) with $a=1$ and $d>\sqrt{2 \lambda}$. Here, g reaches a global minimum at $u=d$ and a local one at $u=0$ while $\mathrm{g}^{\star \star}$ has a unique global minimizer at $u=d$. Then all the local minimizers of $\mathrm{g}$ are not local minimizer for $\mathrm{g}^{\star \star}$. This remark can be easily extended to the $N$ dimensional case of orthogonal matrices since, in this case, replacing the $\ell_{0}$-norm by the CEL0 penalty leads to the convex hull of $\mathrm{G}_{\ell_{0}}$ (showed in $\S 3$ ) which cannot have local minimizers.

In a more general framework, for any matrix $A \in \mathbb{R}^{M \times N}$, let $\hat{\mathrm{x}} \in \mathbb{R}^{N}$ be a local minimizer of $\mathrm{G}_{\ell_{0}}$. Then from Corollary $4.3, \hat{\mathrm{x}}_{\hat{\sigma}}$ solves

$$
\left(A_{\hat{\sigma}}\right)^{T} A_{\hat{\sigma}} \hat{\mathrm{x}}_{\hat{\sigma}}=\left(A_{\hat{\sigma}}\right)^{T} d
$$

where $\hat{\sigma}=\sigma(\hat{\mathrm{x}})$. To be a critical point of $\mathrm{G}_{\text {CELO }}$, such a $\hat{\mathrm{x}}$ has to verify conditions (4.4). Then $\hat{\mathrm{x}}$ is a critical point of $\mathrm{G}_{\text {CELO }}$ if and only if, in addition to solve (4.23), it verifies the following conditions,

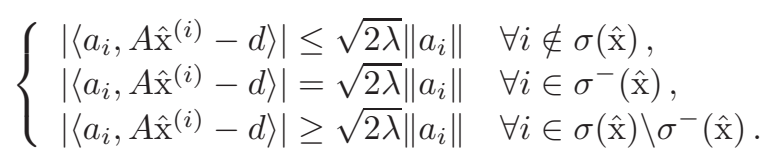

Consequently, if $\hat{\mathrm{x}}$, a local minimizer of $\mathrm{G}_{\ell_{0}}$, does not verify (4.24), it is not a critical point of $\mathrm{G}_{\text {CELO }}$ and then $\mathrm{G}_{\text {CELo }}$ does not admit a local minimum at $\hat{\mathrm{x}}$. Indeed, if $\hat{\mathrm{x}}$ verifies $(4.23)$ then $\forall i \in \sigma(\hat{\mathrm{x}}), \hat{\mathrm{x}}_{i}=$ $-\left\langle a_{i}, A \hat{\mathrm{x}}^{(i)}-d\right\rangle /\left\|a_{i}\right\|^{2}$ and we get,

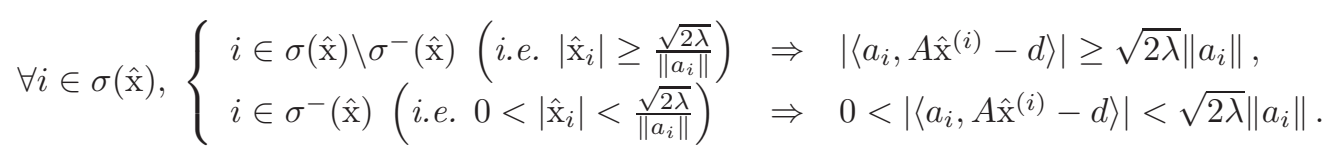

Therefore, a minimizer $\hat{\mathrm{x}}$ of $\mathrm{G}_{\ell_{0}}$ which necessarily verifies (4.23) cannot verify the second line of $(4.24)$ if $\sigma^{-}(\hat{\mathrm{x}}) \neq \emptyset$. Moreover, it is also not ensured to verify the first line of (4.24). Then, some local minimizers of $\mathrm{G}_{\ell_{0}}$ are not critical points of $\mathrm{G}_{\mathrm{CEL} \text { o }}$.

In particular, $\mathrm{G}_{\mathrm{CEL}}$ eliminates the strict local minimizers $\hat{\mathrm{x}}$ of $\mathrm{G}_{\ell_{0}}$ such that $\sigma^{-}(\hat{\mathrm{x}}) \neq \emptyset$. This will be numerically illustrated on low dimensional examples in $\S 4.2$.

A consequence of Lemma 4.4 and Theorem 4.8 is given by the following corollary.

Corollary 4.9. Let $\hat{\mathrm{x}}$ be a strict (local) minimizer of $\mathrm{G}_{\mathrm{CEL}}$, then $\hat{\mathrm{x}}$ is a strict (local) minimizer of $\mathrm{G}_{\ell_{0}}$. Furthermore, $A_{\sigma(\hat{\mathrm{x}})}$ has a full column rank.

Proof. Since $\hat{\mathrm{x}}$ is a strict (local) minimizer of $\mathrm{G}_{\mathrm{CEL} O}$, we get from Lemma 4.4 that $\hat{\mathrm{x}}$ verifies $\sigma^{-}(\hat{\mathrm{x}})=\emptyset$. Then, Theorems 4.5 and 4.8 ensure that it is a (local) minimizer of $\mathrm{G}_{\ell_{0}}$ and that $\mathrm{G}_{\text {CELo }}(\hat{\mathrm{x}})=\mathrm{G}_{\ell_{0}}(\hat{\mathrm{x}})$. Let us now suppose that $\hat{\mathrm{x}}$ is not strict for $\mathrm{G}_{\ell_{0}}$. Then there exists a neighbourhood $\mathcal{V}_{0} \subset \mathbb{R}^{N}$ containing $\hat{\mathrm{x}}$ such that for all $\mathcal{V} \subset \mathcal{V}_{0}$ containing $\hat{\mathrm{x}}$, there exists $\overline{\mathrm{x}} \in \mathcal{V}$ verifying $\mathrm{G}_{\ell_{0}}(\overline{\mathrm{x}})=\mathrm{G}_{\ell_{0}}(\hat{\mathrm{x}})$. Since by definition $\mathrm{G}_{\mathrm{CEL}}$ underestimates $\mathrm{G}_{\ell_{0}}$ (see Remark 4.1) we have

$$
\forall \mathcal{V} \subset \mathcal{V}_{0} \text {, s.t. } \hat{\mathrm{x}} \in \mathcal{V}, \exists \overline{\mathrm{x}} \in \mathcal{V}, \mathrm{G}_{\mathrm{CELO}}(\overline{\mathrm{x}}) \leq \mathrm{G}_{\ell_{0}}(\overline{\mathrm{x}})=\mathrm{G}_{\ell_{0}}(\hat{\mathrm{x}})=\mathrm{G}_{\mathrm{CEL} \mathrm{O}}(\hat{\mathrm{x}}),
$$

which contradicts the fact that $\hat{\mathrm{x}}$ is a strict (local) minimizer of $\mathrm{G}_{\mathrm{CEL} \text { o }}$. Therefore $\hat{\mathrm{x}}$ is a strict (local) minimizer of $\mathrm{G}_{\ell_{0}}$. Then [32, Theorem 3.2] ensures that $\operatorname{rank}\left(A_{\sigma(\hat{\mathrm{x}})}\right)=\# \sigma(\hat{\mathrm{x}})$ and completes the proof.

Finally, in the following proposition we give a characteristic of the function $\mathrm{G}_{\text {CELo }}$ which could be useful to design specific algorithms. 
Proposition 4.10. For $i \in \mathbb{I}_{N}$, the restriction $\mathrm{G}_{\mathrm{CELO}}^{i}$ of $\mathrm{G}_{\mathrm{CEL} O}$ to the ith variable is affine on $\left(-\frac{\sqrt{2 \lambda}}{\left\|a_{i}\right\|}, 0\right) \cup$ $\left(0, \frac{\sqrt{2 \lambda}}{\left\|a_{i}\right\|}\right)$ and strictly convex beyond.

Proof. Let $i \in \mathbb{I}_{N}$ be fixed. Then $\mathrm{G}_{\text {cELo }}^{i}$, the restriction of $\mathrm{G}_{\text {CELo }}$ to the ith variable, can be written as, $\forall t \in \mathbb{R}$

$$
\begin{aligned}
\mathrm{G}_{\text {cELo }}^{i}(t) & =\mathrm{G}_{\mathrm{CELO}}\left(\mathrm{x}^{(i)}+t e_{i}\right) \\
& =\frac{1}{2}\left\|A \mathrm{x}^{(i)}+a_{i} t-d\right\|^{2}+\sum_{j \in \mathbb{I}_{N} \backslash\{i\}} \phi\left(\left\|a_{j}\right\|, \lambda ; \mathrm{x}_{j}\right)+\phi\left(\left\|a_{i}\right\|, \lambda ; t\right) \\
& =\frac{1}{2}\left\|A \mathrm{x}^{(i)}-d\right\|^{2}+t\left\langle a_{i}, A \mathrm{x}^{(i)}-d\right\rangle+\frac{1}{2}\left\|a_{i}\right\|^{2} t^{2}+\sum_{j \in \mathbb{I}_{N} \backslash\{i\}} \phi\left(\left\|a_{j}\right\|, \lambda ; \mathrm{x}_{j}\right)+\phi\left(\left\|a_{i}\right\|, \lambda ; t\right) \\
& =\underbrace{\frac{1}{2}\left\|A \mathrm{x}^{(i)}-d\right\|^{2}+\sum_{j \in \mathbb{I}_{N} \backslash\{i\}} \phi\left(\left\|a_{j}\right\|, \lambda ; \mathrm{x}_{j}\right)-\frac{(\tilde{d})^{2}}{2}}_{\text {Independant of } \mathrm{t}}+\frac{1}{2}\left(\left\|a_{i}\right\| t-\tilde{d}\right)^{2}+\phi\left(\left\|a_{i}\right\|, \lambda ; t\right),
\end{aligned}
$$

where $\tilde{d}=\left\langle a_{i}, d-A \mathrm{x}^{(i)}\right\rangle /\left\|a_{i}\right\| \in \mathbb{R}$. Then with (2.8) the result is straightforward.

\subsection{Numerical illustrations of the reciprocal of Theorem 4.8}

In this part, we experimentally study the fact that $\mathrm{G}_{\text {CELo }}$ has "less" strict local minima than $\mathrm{G}_{\ell_{0}}$.

\subsubsection{Two dimensional examples}

Here, we consider the case $N=M=2$. On Figure 2 we present the isolevels of both $\mathrm{G}_{\ell_{0}}$ and $\mathrm{G}_{\text {СЕLо }}$ for different $A \in \mathbb{R}^{2 \times 2}, d \in \mathbb{R}^{2}$ and $\lambda>0$. In all cases, global minimizers of $\mathrm{G}_{\ell_{0}}$ are also global minimizers of $\mathrm{G}_{\text {CELo }}$ as stated by Theorem 4.5. For the example given in $2(\mathrm{a})$ and $2(\mathrm{~b}), \forall \mathrm{x}^{\star} \in[0,1]^{2}, \mathrm{x}^{\star}$ is a global minimizer of $\mathrm{G}_{\text {CELO }}$. Let $\left.\hat{\mathrm{x}} \in\right] 0,1\left[^{2}\right.$, which is thus a global optimum of $\mathrm{G}_{\mathrm{CELO}}$, then it is clear on this example that $\hat{x}^{0}$ defined by (4.13) is a global minimizer for both $G_{\text {CЕLо }}$ and $G_{\ell_{0}}$ as stated by the second point of Theorem 4.5. This example also illustrates the Remark 4.5.

Other examples of Figure 2 illustrate that $\mathrm{G}_{\text {СЕLо }}$ has in general less local minima than $\mathrm{G}_{\ell_{0}}$. When $A \in \mathbb{R}^{2 \times 2}, \mathrm{G}_{\ell_{0}}$ has four minima (local or global). On the example presented by Figures $2(\mathrm{c})$ and $2(\mathrm{~d})$ we can see that $G_{\text {CEL }}$ has only two global minima and when we consider the example given on Figures 2(e) and $2(\mathrm{f}), \mathrm{G}_{\mathrm{CEL}}$ has an unique global minimum. For these two examples the use of the CEL0 penalty allows to eliminate all the local minimizers of $\mathrm{G}_{\ell_{0}}$ while the global ones are preserved. However this is not always the case as we can see on the example of Figures $2(\mathrm{~g})$ and $2(\mathrm{~h})$ where $\mathrm{G}_{\text {СЕо }}$ has one global and one local minimizer. In this example $\mathrm{G}_{\mathrm{CEL}}$ has two local minimizers less than $\mathrm{G}_{\ell_{0}}$.

\subsubsection{For an arbitrary dimension}

Let us now consider examples in higher dimension. Following the numerical illustrations of [32, §6.2], we define $\mathrm{G}_{\ell_{0}}$ with $M=5$ and $N=10$ using

$$
A=\left(\begin{array}{llllllllll}
7 & 2 & 4 & 9 & 0 & 3 & 3 & 6 & 6 & 7 \\
3 & 4 & 9 & 3 & 3 & 9 & 1 & 3 & 1 & 5 \\
5 & 4 & 2 & 4 & 0 & 7 & 1 & 9 & 2 & 9 \\
8 & 4 & 0 & 9 & 6 & 0 & 4 & 2 & 3 & 7 \\
6 & 3 & 6 & 5 & 0 & 9 & 0 & 0 & 3 & 8
\end{array}\right)
$$




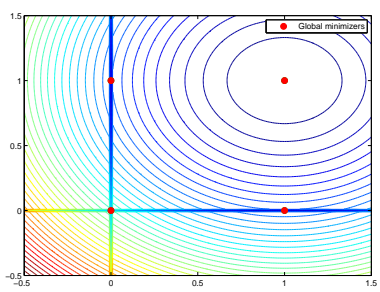

(a) $G_{\ell_{0}}$

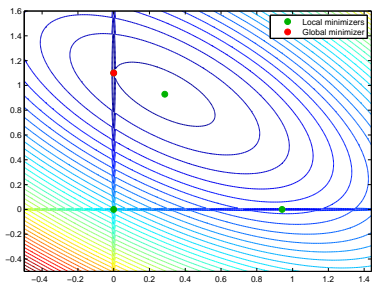

(e) $G_{\ell_{0}}$

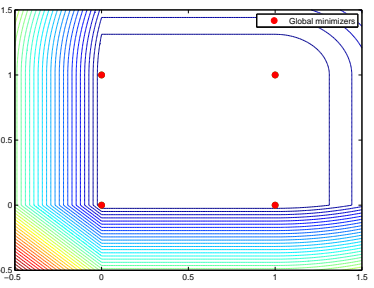

(b) $\mathrm{G}_{\mathrm{CELO}}$

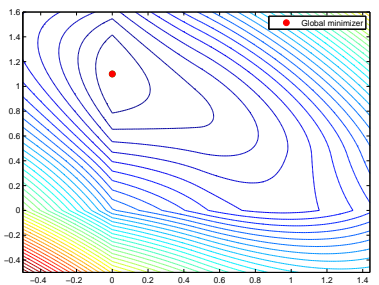

(f) $\mathrm{G}_{\mathrm{CELO}}$

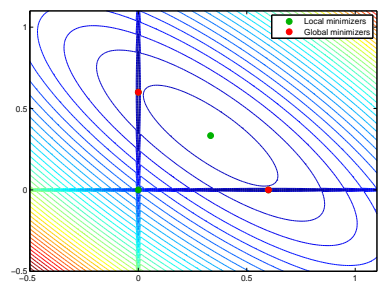

(c) $G_{\ell_{0}}$

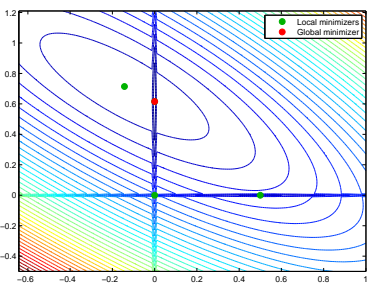

(g) $\mathrm{G}_{\ell_{0}}$

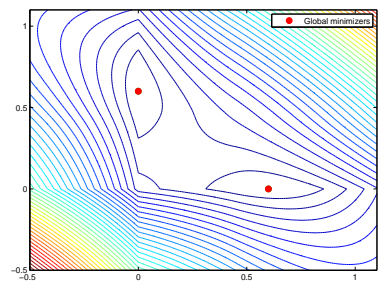

(d) $\mathrm{G}_{\mathrm{CELO}}$

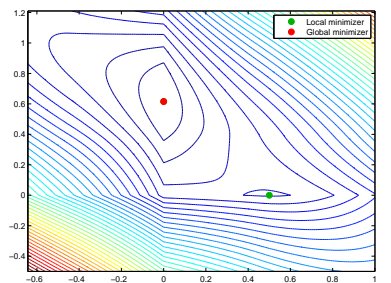

(h) $\mathrm{G}_{\text {CELO }}$

Figure 2: Isolevels of $\mathrm{G}_{\ell_{0}}$ and $\mathrm{G}_{\text {CELO }}$ for four examples where $N=M=2$. (a)-(b) $A=[1,0 ; 0,1]$, $b=[1 ; 1]$ and $\lambda=0.5$ (c)-(d) $A=[1,2 ; 2,1], b=[1 ; 1]$ and $\lambda=0.5$ (e)-(f) $A=[0.5,2 ; 2,1], b=[2 ; 1.5]$ and $\lambda=0.5(\mathrm{~g})-(\mathrm{h}) A=[3,2 ; 1,3], b=[1 ; 2]$ and $\lambda=1$. Green points are local minimizers and red ones are global.

and

$$
d=A \mathrm{x}^{\star} \text { with } \mathrm{x}^{\star}=(0,1,8,0,3,0,0,0,0,9)^{T} .
$$

Then, we will study the strict (local) minimizers of $\mathrm{G}_{\ell_{0}}$ and $\mathrm{G}_{\mathrm{CEL} \text { o }}$. As stated by [32, Theorem 4.4], the global minimizers of $\mathrm{G}_{\ell_{0}}$ are strict. That makes strict minimizers interesting. Following [32, Corollary 3.3] a strict (local) minimizer of $\mathrm{G}_{\ell_{0}}$ can be easily computed by choosing a support $\omega \in \Omega_{\max }$, where $\Omega_{\max }$ is defined by [32, Definition 3.1]

$$
\Omega_{\max }=\bigcup_{r=0}^{M} \Omega_{r} \text { where } \Omega_{r}=\left\{\omega \subset \mathbb{I}_{N}: \# \omega=r=\operatorname{rank}\left(A_{\omega}\right)\right\}
$$

and then solve the normal equations restricted to this support (as in (4.8)). In other words, strict (local) minimizers are the ones with a support belonging to $\Omega_{\max }$.

Then one can compute all strict (local) minimizers by solving the restricted normal equations for all the supports of $\Omega_{\max }$ which is a finite set. This has been done for $\mathrm{G}_{\ell_{0}}$ defined according to (4.26) and (4.27). Results have been displayed on Figure 3(a) in the same way as in [32] where the x-axis lists all the strict (local) minimizers according to their support length and the y-axis gives the associated value of $\mathrm{G}_{\ell_{0}}$. Among all these 638 (local) minimizers we can keep only those verifying (4.24) which are critical points of $\mathrm{G}_{\mathrm{CEL} \mathrm{O}}$. They are represented on Figure 3(c). Only 283 of the 638 (local) minimizers of $\mathrm{G}_{\ell_{0}}$ are thus preserved. Following Corollary 4.9 these 283 critical points of $\mathrm{G}_{\text {CEL }}$ contain all the strict (local) minimizers of $\mathrm{G}_{\mathrm{CEL} 0}$. Indeed suppose that $\hat{\mathrm{x}} \in \mathbb{R}^{N}$, a strict (local) minimizer of $\mathrm{G}_{\mathrm{CEL} \text { o }}$, is not represented on Figure 3(c). Then by construction it is neither represented on Figure 3(a). But, according to Corollary 4.9 , it is also a strict (local) minimizer of $\mathrm{G}_{\ell_{0}}$. This contradict the fact that Figure 3(a) contains all strict (local) minimizers of $\mathrm{G}_{\ell_{0}}$ and prove the previous affirmation. However strict minimizers of $\mathrm{G}_{\ell_{0}}$ which are preserved on Figure 3(c) are not necessary minimizers for $\mathrm{G}_{\text {CEL。 }}$ (we only know that they are critical points for $\mathrm{G}_{\text {CELO }}$ ) and those which are minimizers for $\mathrm{G}_{\text {CELO }}$ are not 
necessarily strict. This is illustrated with the global minima of the example given on Figures 2(a) and 2 (b) which are strict for $\mathrm{G}_{\ell_{0}}$ and not strict for $\mathrm{G}_{\mathrm{CEL} O}$.

Using the CEL0 penalty instead of the $\ell_{0}$-norm seems to remove a significant number of strict local minimizers of $\mathrm{G}_{\ell_{0}}$. This is interesting since algorithms will have a "lower probability" to get trapped into a local minimizer. We can also observe on the zooms $3(\mathrm{~b})$ and $3(\mathrm{~d})$ that the unique global minimizer (see [32] for the uniqueness) of $\mathrm{G}_{\ell_{0}}$ is preserved which illustrates Theorem 4.5. Finally one can see that among all the strict local minimizers of $G_{\ell_{0}}, G_{\text {CEL }}$ seems to preserve the ones with the lower value of $\mathrm{G}_{\ell_{0}}$ (or $\mathrm{G}_{\text {CЕL }}$ since we have equality for these points).

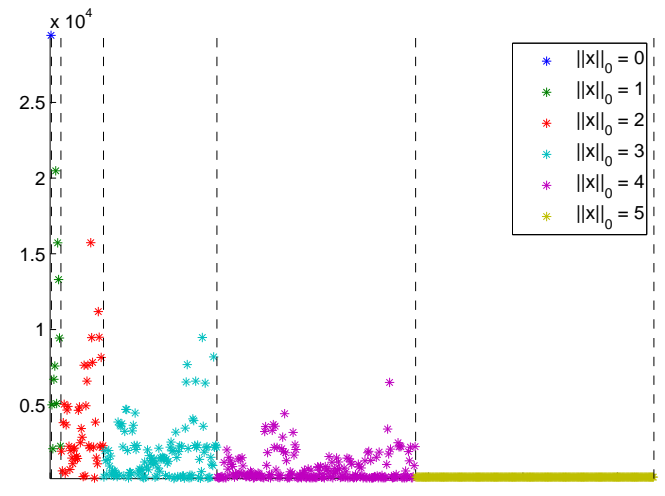

(a) All 638 strict (local) minima of $\mathrm{G}_{\ell_{0}}$

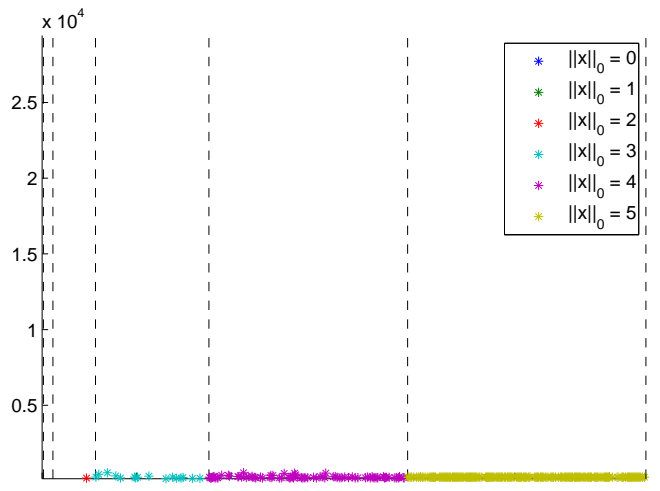

(c) The $\mathbf{2 8 3}$ which are critical points of $\mathrm{G}_{\mathrm{CELO}}$

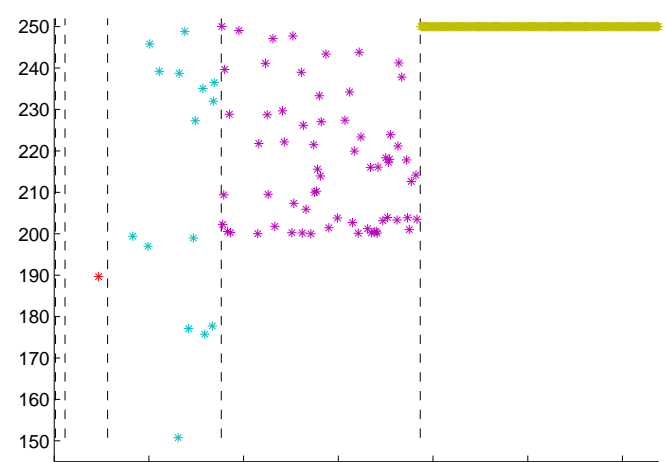

(b) Zoom of 3(a) along the y-axis

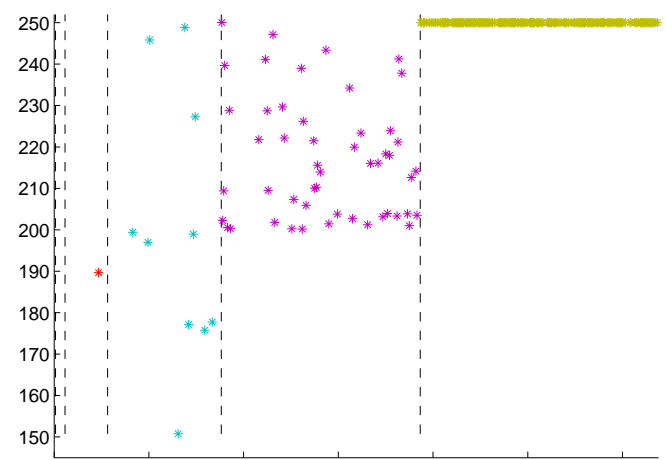

(d) Zoom of 3(c) along the y-axis

Figure 3: (a)-(b) Strict (local) minima of $\mathrm{G}_{\ell_{0}}$ defined according to equations (4.26) and (4.27) for $\lambda=50$. (c)-(d) The ones verifying (4.24) which are critical points of $\mathrm{G}_{\text {CELO }}$.

In Table 1 we have computed the number of strict (local) minimizers of $\mathrm{G}_{\ell_{0}}$ for different values of $M$ and $N$. For each couple $(M, N), 1000$ random matrices $A \in \mathbb{R}^{M \times N}$ and $d \in \mathbb{R}^{M}$ have been generated from an uniform distribution and the number of strict (local) minimizers of $\mathrm{G}_{\ell_{0}}$ preserved with $\mathrm{G}_{\text {cЕLo }}$ (we denote this number by $\mathrm{P}$ ) has been determined. We report in Table 1 the minimal, maximal and mean values of $\mathrm{P}$ with respect to the 1000 realizations. These experiments are in agreement with the fact that $\mathrm{G}_{\text {сЕLо }}$ has significantly less strict local minimizers than $\mathrm{G}_{\ell_{0}}{ }^{2}$. We can also remark, in Table 1 ,

\footnotetext{
${ }^{2}$ Theorem 4.5 ensures that removed minimizers from $\mathrm{G}_{\ell_{0}}$ to $\mathrm{G}_{\mathrm{CEL}}$ are not global.
} 
that the number of removed strict minimizers with $\mathrm{G}_{\text {CELO }}$ is higher with a larger value of $\lambda$.

Table 1: Number of strict (local) minimizers $\left(\# \Omega_{\max }\right)$ of $\mathrm{G}_{\ell_{0}}$ and the number $P$ of them which are critical points of $\mathrm{G}_{\text {CELO }}$. This is computed for 1000 matrices with entries randomly generated from an uniform distribution. $\mathrm{P}_{\min }, \mathrm{P}_{\max }$ and $\overline{\mathrm{P}}$ correspond respectively to the minimal, the maximal and the mean value of $\mathrm{P}$ over the 1000 realizations. This experiment is repeated for different matrix sizes and two different $\lambda$.

\begin{tabular}{|c|c||c|c|c|c|}
\hline & & $\# \Omega_{\max }$ & $\mathrm{P}_{\min }$ & $\mathrm{P}_{\max }$ & $\overline{\mathrm{P}}$ \\
\hline \hline \multirow{3}{*}{$\lambda=0.5$} & $M=3, N=5$ & 26 & 1 & 20 & 9 \\
\cline { 2 - 6 } & $M=5, N=10$ & 638 & 39 & 347 & 206 \\
\cline { 2 - 6 } & $M=7, N=15$ & 16384 & 2237 & 7374 & 5037 \\
\hline \hline \multirow{3}{*}{$\lambda=0.1$} & $M=3, N=5$ & 26 & 4 & 23 & 13 \\
\cline { 2 - 6 } & $M=5, N=10$ & 638 & 173 & 444 & 306 \\
\cline { 2 - 6 } & $M=7, N=15$ & 16384 & 5370 & 9431 & 7570 \\
\hline
\end{tabular}

\subsection{The case where $A$ is orthogonal}

As it has been shown in $\S 3$, when $A$ is orthogonal, $\mathrm{G}_{\text {CELO }}$ is the convex hull of $\mathrm{G}_{\ell_{0}}$. Thus $\mathrm{G}_{\text {CELo }}$ is convex and all its critical points are global minimizers of $\mathrm{G}_{\text {CEL }}$ from which, following Theorem 4.5, we can deduce global minimizers of $\mathrm{G}_{\ell_{0}}$. Using the orthogonality of $A$ we can rewrite the critical point characterization (4.4) given by Lemma 4.1 as follows

$$
\forall i \in \mathbb{I}_{N}, \begin{cases}\hat{\mathrm{x}}_{i}=0 & \text { if }\left|\left\langle a_{i}, d\right\rangle\right| \leq \sqrt{2 \lambda}\left\|a_{i}\right\|, \\ \hat{\mathrm{x}}_{i}=-s_{i} t, t \in\left[0, \frac{\sqrt{2 \lambda}}{\left\|a_{i}\right\|}\right] & \text { if }\left|\left\langle a_{i}, d\right\rangle\right|=\sqrt{2 \lambda}\left\|a_{i}\right\|, \\ \hat{\mathrm{x}}_{i}=\frac{\left\langle a_{i}, d\right\rangle}{\left\|a_{i}\right\|^{2}} & \text { if }\left|\left\langle a_{i}, d\right\rangle\right| \geq \sqrt{2 \lambda}\left\|a_{i}\right\| .\end{cases}
$$

Let $z=A^{T} d$, then the sparsest minimizer of $\mathrm{G}_{\text {CELO }}$, denoted $\hat{\mathrm{x}}^{0}$, is given by the following thresholding rule

$$
\forall i \in \mathbb{I}_{N}, \hat{\mathrm{x}}_{i}^{0}=\frac{z_{i}}{\left\|a_{i}\right\|^{2}} \mathbb{1}_{\left\{\left|z_{i}\right|>\sqrt{2 \lambda}\left\|a_{i}\right\|\right\}} .
$$

Finally, when $A$ is orthogonal, replacing the $\ell_{0}$-norm by the CEL0 penalty transforms the initial problem into a convex problem (its convex envelop) for which we have an analytic expression (4.30) of the sparsest solution. Note that in (4.30) the threshold is different for each components $\mathrm{x}_{i}\left(i \in \mathbb{I}_{N}\right)$ and involves the norm of the columns of $A$. When the columns of $A$ are normalized $\left(i . e .\left\|a_{i}\right\|=1, \forall i \in \mathbb{I}_{N}\right.$ ) we get the well known hard thresholding rule.

\subsection{Conclusions about the CEL0 penalty}

In this section, we made an analytical study of the Continuous Exact $\ell_{0}$ penalty obtained previously by computing the convex hull of $\mathrm{G}_{\ell_{0}}$ in the case where $A$ is orthogonal. It leads to a tight continuous relaxation of the objective function $\mathrm{G}_{\ell_{0}}$ in (1.4), denoted by $\mathrm{G}_{\text {CELO }}$. Global minimizers of $\mathrm{G}_{\text {CELO }}$ contain those of $\mathrm{G}_{\ell_{0}}$ and conversely, from each global minimizer of $\mathrm{G}_{\mathrm{CEL}}$, one can easily extract with (4.13) a global minimizer of $\mathrm{G}_{\ell_{0}}$ (Theorem 4.5). Then Theorem 4.8 extends the second point of this result to local minimizers: from all local minimizers of $\mathrm{G}_{\text {CELo }}$, (4.13) provides a local minimizer of $\mathrm{G}_{\ell_{0}}$. We showed that the converse is false and that experimentally, a large number of strict local minimizers of $\mathrm{G}_{\ell_{0}}$ are not critical points of $\mathrm{G}_{\mathrm{CEL} O}$. In particular $\mathrm{G}_{\mathrm{CEL} 0}$ eliminates the local minimizers $\hat{\mathrm{x}}$ of $\mathrm{G}_{\ell_{0}}$ such that 
$\sigma^{-}(\hat{\mathrm{x}}) \neq \emptyset$. We also have observed that local minimizers of $\mathrm{G}_{\ell_{0}}$ which are not critical points of $\mathrm{G}_{\text {cELo }}$ seem to be the ones corresponding to the higher values of $\mathrm{G}_{\ell_{0}}$ (among all the strict local minimizers of $\left.\mathrm{G}_{\ell_{0}}\right)$. Although $\mathrm{G}_{\text {CEL }}$ remains nonconvex, it is convex with respect to each variable and its continuity offers the ability to use recent nonsmooth nonconvex algorithms to deal with the $\ell_{2}-\ell_{0}$ problem as we will see in the following paragraph.

\section{Algorithms to deal with the $\ell_{2}-$ CEL0 problem}

The analysis provided in the previous section on the link between the $\ell_{2}-\ell_{0}$ and $\ell_{2}$-CEL0 problems allows us to address problem (1.4) by minimizing $\mathrm{G}_{\text {СEL }}$ which has the nice property to be continuous unlike $\mathrm{G}_{\ell_{0}}$. Numerous recent algorithms have been dedicated to such nonsmooth nonconvex functionals. Let us mention three of them which are well adapted to the minimization of $\mathrm{G}_{\mathrm{CEL} 0}$. In the framework of Difference of Convex (DC) functions programming, Gasso et al [20] propose a generic algorithm for solving problem (1.4) where the $\ell_{0}$-norm is replaced by a nonconvex penalty belonging to a particular family of functions. Note that the CEL0 penalty belongs to this family. They propose a suitable decomposition for these penalties as the difference of two convex functions to formulate the problem as a DC program. In this particular decomposition, one function is the $\ell_{1}$-norm which allows them to use efficient schemes to solve each step of the DC algorithm. A decrease of the cost function and a convergence to a critical point is ensured by [20, Theorem 1]. More recently, Ochs et al [34] study a sub-class of Majorization-Minimization (MM) algorithms to minimize energies belonging to a family of nonsmooth nonconvex functions containing in particular $\mathrm{G}_{\mathrm{CEL}}$. Under the Kurdyka-Lojasiewicz (KL) property of the objective function, they prove the convergence of the generated sequence to a critical point by showing that all the requirement of [1, Theorem 2.9] hold. They detail four particular instances of this general sub-class of algorithms. Among them, the Iterative Reweighted $\ell_{1}$ algorithm (IRL1) is the more appropriated for the minimization of our objective function $\mathrm{G}_{\mathrm{CEL}}$ since it majorizes the function using tangents. Such a majorization is well adapted to approximate the singularity of the CEL0 penalty at zero. An experimental analysis shows the ability of the algorithm to avoid local minimizers on some examples. Whenever this can not be generalized, it illustrates an interesting behaviour of the proposed algorithm. Finally we can cite the General Iterative Shrinkage and Thresholding (GIST) algorithm proposed by Gong et al [22] to solve nonconvex penalized problems. It deals with a large class of nonconvex penalties including CEL0. GIST is an iterative forward-backward splitting algorithm combined with a line search to select a good step size a each iteration. Convergence to a critical point of the objective function is proved. Like the algorithm proposed by Ochs et al, GIST belongs to the family of MM algorithms. One can see this algorithm as an extension of the Iterative Hard Thresholding to other nonconvex penalties than the $\ell_{0}$-norm. In a recent work, Kowalski [23] shows the convergence of ISTA with any thresholding rule which leads to similar numerical schemes as GIST.

Note that all the mentioned algorithms have been proposed during the last years and this is not an exhaustive list. Optimization of such nonsmooth nonconvex functionals is thus a very active research topic which suggests that new improvements will be done in the next years.

\subsection{A macro algorithm to find (local) minimizers of $G_{\ell_{0}}$}

Let $\operatorname{Alg}\left(\mathrm{x}^{\text {init }}, \lambda\right)$ be an algorithm, initialized with $\mathrm{x}^{\text {init }} \in \mathbb{R}^{N}$ (first guess) and $\lambda>0$ (hyperparameter), producing a sequence of iterates $\left(\mathrm{x}^{k}\right)_{k \in \mathbb{N}}$ which

H1. converges to a critical point of $\mathrm{G}_{\mathrm{CEL}}$,

$\mathrm{H} 2$. verifies

$$
\forall k \in \mathbb{N}, \mathrm{G}_{\mathrm{CELO}}\left(\mathrm{x}^{k+1}\right) \leq \mathrm{G}_{\text {CELO }}\left(\mathrm{x}^{k}\right)-\beta\left\|\mathrm{x}^{k+1}-\mathrm{x}^{k}\right\|^{2}
$$


where $\beta$ is a positive constant.

Condition $\mathrm{H} 2$ is also called sufficient-decrease condition. It is usually used to prove convergence of descent algorithms. In the following, we denote $\sigma_{k}^{-}=\sigma^{-}\left(\mathrm{x}^{k}\right)$.

Based on such an algorithm Alg, for example one of the algorithms mentioned above, we propose the "macro" algorithm detailed in Algorithm 1. The idea of this macro algorithm is to move iteratively from a critical point of $\mathrm{G}_{\text {CEL }}$ to another one, decreasing the cost function, until reaching a critical point of $\mathrm{G}_{\mathrm{CEL}}, \hat{\mathrm{x}}$, verifying $\sigma^{-}(\hat{\mathrm{x}})=\emptyset$. From Lemma 4.7 , such a critical point is a (local) minimizer of $\mathrm{G}_{\ell_{0}}$.

Remark 5.1. In view of Remark 4.5, one can also define $\sigma_{k}^{-}:=\sigma^{-}\left(\mathrm{x}^{k}\right) \cup\left\{i \in \mathbb{I}_{N}:\left|\mathrm{x}_{i}^{k}\right|=\frac{\sqrt{2 \lambda}}{\left\|a_{i}\right\|}\right\}$. This will allow to set more entries of $\mathrm{x}^{k}$ to zero.

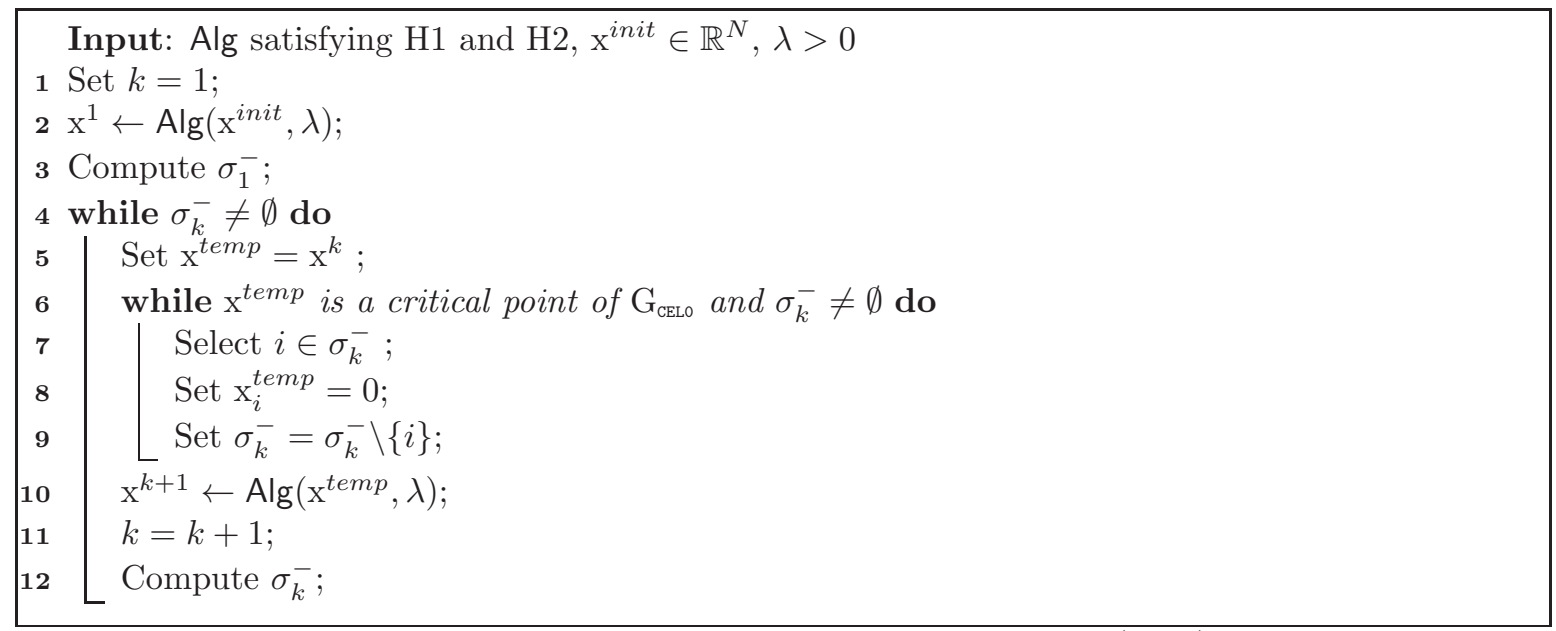

Algorithm 1: A macro algorithm to find critical points of $G_{\text {CELo }}$ being (local) minimizer for $G_{\ell_{0}}$.

Starting with a critical point $\mathrm{x}^{1}$ of $\mathrm{G}_{\text {CELo }}$ obtained by Alg (line 2), Algorithm 1 sets (at iteration $k$ ) one by one the components indexed by $i \in \sigma_{k}^{-}$to zero while the resulting point (defined by line 8 ) remains a critical point of $\mathrm{G}_{\text {CELo }}$. If all the components belonging to $\sigma_{k}^{-}$are set to zero then the algorithm stops and returns this new point which is a critical point of $\mathrm{G}_{\mathrm{CEL} o}$ verifying $\sigma_{k}^{-}=\emptyset$. Otherwise if the setting of one $\mathrm{x}_{i}^{k}, i \in \sigma_{k}^{-}$, to zero results in a point which is not a critical point of $\mathrm{G}_{\text {CELo }}$, the next iterate $\mathrm{x}^{k+1}$ is computed by Alg initialized with this non stationary point (line 10). Following the hypothesis $\mathrm{H} 1$ on $\mathrm{Alg}, \mathrm{x}^{k+1}$ is a critical point of $\mathrm{G}_{\mathrm{CELO}}$.

Note that the algorithm is ensured to stop since from all global minimizer $\hat{\mathrm{x}} \in \mathbb{R}^{N}$ of $\mathrm{G}_{\text {CELo }}$ (there exists at least one from Proposition 4.6)), Theorem 4.5 allows to define another global optimum $\hat{\mathrm{x}}^{0}$ (defined by (4.13)) verifying $\sigma^{-}\left(\hat{\mathrm{x}}^{0}\right)=\emptyset$. Therefore the set of critical points $\hat{\mathrm{x}}$ of $\mathrm{G}_{\text {CELo }}$ verifying $\sigma^{-}(\hat{\mathrm{x}})=\emptyset$ is nonempty. The following theorem gives a more formal convergence result on the generated sequence $\left(\mathrm{x}^{k}\right)_{k \in \mathbb{N}}$.

Theorem 5.1 (Convergence of the macro algorithm). Let $\left(\mathrm{x}^{k}\right)_{k \in \mathbb{N}}$ be the sequence generated by Algorithm 1. There exists $k^{\star} \in \mathbb{N}$ such that $\sigma_{k^{\star}}^{-}=\emptyset$ and $\mathrm{x}^{k^{\star}}$ is a (local) minimizer of $\mathrm{G}_{\ell_{0}}$.

Proof. The proof is given in Appendix D. 
Remark 5.2. The proof of Theorem 5.1, detailed in Appendix D, is based on the fact that the image of the set of Clarke critical points of $\mathrm{G}_{\mathrm{CEL}}$ is finite. The number of elements of this set can be huge which suggests that the macro algorithm can stop after a large number of iteration $k^{\star} \in \mathbb{N}$. However in the numerical examples presented in $§ 5.3$, the macro algorithm generally stops after one or two iterations. Indeed, performing many iterates in the outer loop of Algorithm 1 means that Alg reaches "unstable" critical points (i.e. which are not minimizers). Even if Alg can converge to such points, it is less frequent than getting trapped into a (local) minimizer.

Remark 5.3. The selection at line 7 is arbitrary but one can imagine to find an "optimal"- strategy for this selection.

\subsection{Note on the Iterative Hard Thresholding (IHT) algorithm}

Introduced by Blumensath and Davies in [4], the IHT algorithm performs iterates as

$$
\mathrm{x}^{k+1}=H_{\lambda}\left(\mathrm{x}^{k}-A^{T}(A \mathrm{x}-d)\right)
$$

where $H_{\lambda}\left(\mathrm{x}_{i}\right)=\mathrm{x}_{i} \mathbb{1}_{\left\{\left|\mathrm{x}_{i}\right|>\sqrt{2 \lambda}\right\}}$ denotes the hard thresholding function. It has been proved to converge to a (local) minimizer of $\mathrm{G}_{\ell_{0}}$ under the condition that $\|A\|<1$ [4]. More recently some authors showed that the convergence of this forward-backward splitting algorithm actually holds for any matrix $A \in \mathbb{R}^{M \times N}$ [1] considering a slightly different scheme from (5.2) including an additional step size $\gamma^{k}$ (such that $\sup _{k} \gamma^{k}<1 /\|A\|^{2}$ ),

$$
\mathrm{x}^{k+1}=H_{\lambda \gamma^{k}}\left(\mathrm{x}^{k}-\gamma^{k} A^{T}(A \mathrm{x}-d)\right)
$$

Using such a forward-backward iteration [1] on $\mathrm{G}_{\text {CEL }}$ gives,

$$
\mathrm{x}^{k+1} \in \operatorname{prox}_{\gamma^{k} \Phi \text { CELo }(\cdot)}\left(\mathrm{x}^{k}-\gamma^{k} A^{T}\left(A \mathrm{x}^{k}-d\right)\right)
$$

where $0<\underline{\gamma}<\gamma^{k}<\bar{\gamma}<\frac{1}{L}$ with $L=\frac{1}{\|A\|^{2}}$ the Lipschitz constant of the gradient of the quadratic term and prox denotes the proximity operator defined by,

$$
\operatorname{prox}_{\gamma f(\cdot)}(\mathrm{y}):=\underset{\mathrm{x} \in \mathbb{R}^{N}}{\arg \min } \frac{1}{2 \gamma}\|\mathrm{x}-\mathrm{y}\|^{2}+f(\mathrm{x}) .
$$

Since $\Phi_{\text {cELo }}$ is additively separable we obtain that for $\mathrm{y}=\left(\mathrm{y}_{1}, \cdots, \mathrm{y}_{N}\right) \in \mathbb{R}^{N}$

$$
\operatorname{prox}_{\gamma \Phi \mathrm{CELO}(\cdot)}(\mathrm{y})=\left(\operatorname{prox}_{\gamma \phi\left(\left\|a_{1}\right\|, \lambda ; \cdot\right)}\left(\mathrm{y}_{1}\right), \cdots, \operatorname{prox}_{\gamma \phi\left(\left\|a_{N}\right\|, \lambda ; \cdot\right)}\left(\mathrm{y}_{N}\right)\right)
$$

where for $a>0, \lambda>0$ and $\gamma>0$

$$
\operatorname{prox}_{\gamma \phi(a, \lambda ; \cdot)}(u)= \begin{cases}\operatorname{sign}(u) \min \left(|u|,(|u|-\sqrt{2 \lambda} \gamma a)_{+} /\left(1-a^{2} \gamma\right)\right) & \text { if } a^{2} \gamma<1 \\ u \mathbb{1}_{\{|u|>\sqrt{2 \gamma \lambda}\}}+\{0, u\} \mathbb{1}_{\{|u|=\sqrt{2 \gamma \lambda}\}} & \text { if } a^{2} \gamma \geq 1\end{cases}
$$

Following [1], since $\frac{1}{2}\|A \mathrm{x}-d\|^{2}$ is a polynomial function and $\Phi_{\text {CELo }}$ has a piecewise polynomial graph, $\mathrm{G}_{\text {CELo }}$ is semi-algebraic and then verifies the Kurdyka-Lojaseiwicz (KL) property. Therefore $\mathrm{G}_{\text {CELO }}$ is a proper lower semicontinuous KL function bounded from below and [1, Theorem 5.1] ensures the convergence of the sequence $\left(\mathrm{x}^{k}\right)_{k \in \mathbb{N}}$ generated by the scheme (5.4) to a critical point of $\mathrm{G}_{\mathrm{CEL} \text { o }}$. One can notice that,

$$
\|A\|=\sup _{\|\mathrm{x}\|=1}\|A \mathrm{x}\| \geq \max _{i \in \mathbb{I}_{N}}\left\|a_{i}\right\|
$$


which implies that,

$$
\forall k, \forall i \in \mathbb{I}_{N},\left\|a_{i}\right\|^{2} \gamma^{k}<1,
$$

since $\forall k, \gamma^{k}<1 /\|A\|^{2} \leq 1 / \max _{i \in \mathbb{I}_{N}}\left\|a_{i}\right\|^{2}$. Then, the proximity operator of $\phi$ is reduced to the first line of (5.7) which define a continuous threshold function. This thresholding rule differs between each component depending on the norm of the associated column of $A$. Thus, according to our numerical illustrations in $\S 4.2$, some local minimizers of $\mathrm{G}_{\ell_{0}}$ can be avoided using the thresholding rule ((5.7) first line) instead of the hard one $H_{\gamma \lambda}$ in the FBS algorithm.

\subsection{Numerical illustrations}

In this section, we illustrate on low dimensional examples the behaviour of the proposed macro algorithm (Algorithm 1) and compare its performances with the IHT algorithm (in its version defined in [1]). For the macro algorithm, we will consider as inner algorithm Alg, the IRL1 algorithm [34] and the Forward-Backward splitting (FB) defined by the scheme (5.4).

Figure 4 presents the behaviour of the proposed macro algorithm on a 2D example. Red crosses correspond to the inner iterates of Alg while green points are the outer ones. The green cross represent the temporary variable $\mathrm{x}^{t e m p}$ in Algorithm 1. Since Algorithm 1 minimizes $\mathrm{G}_{\mathrm{CEL}}$, these iterates are represented with the isolevels of $\mathrm{G}_{\mathrm{CEL} \text { o }}$. When the IRl1 algorithm is used (Figure 4(a)), the macro algorithm (green points) converges in one iteration to the global minimizer. With the same starting point, the FB algorithm converges to an "unstable" critical point of $\mathrm{G}_{\text {cELo }}$ such that $\sigma^{-}(\hat{\mathrm{x}})=\{1,2\}$ (i.e. the two components of $\hat{\mathrm{x}}$ belongs to $\left.\sigma^{-}(\hat{\mathrm{x}})\right)$. Then, in this case, the macro algorithm performs an outer iteration. On Figures 4(b) and 4(c) we can see that, depending on the selected component (line 7 of Algorithm 1), the macro algorithm converges either to the global minimizer or to a local one. This reflects remark 5.3 on the way to define an "optimal" selection leading to the better (local) minimizer (in terms of minimization).

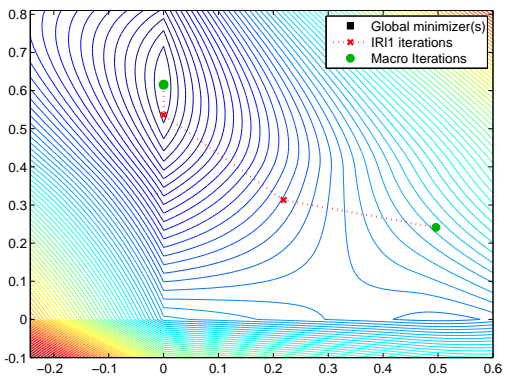

(a) Algorithm 1 with IRl1

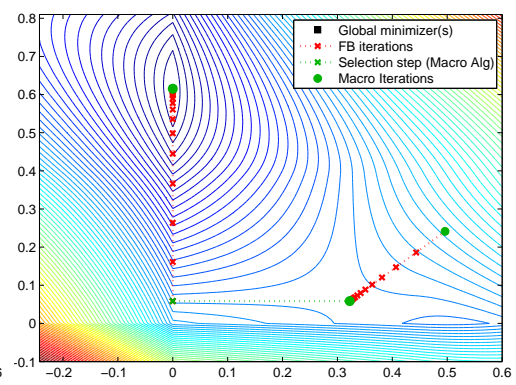

(b) Algorithm 1 with FB

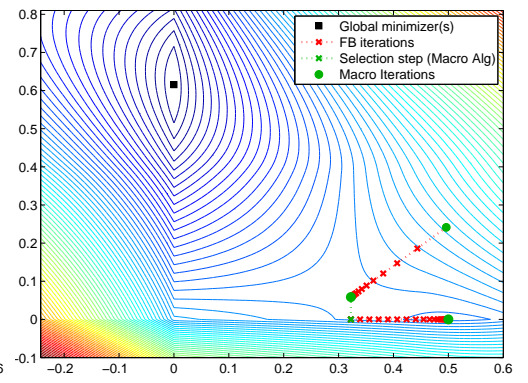

(c) Algorithm 1 with FB

Figure 4: Iterates evolution of Algorithm 1 on the example defined by Figure 2(h). The difference between (b) and (c) holds in the selection process of line 7 in Algorithm 1.

Note that on the examples of Figure 4, the starting point has been defined in order to have two iterates in the outer loop of the macro algorithm with FB. This illustrates remark 5.2 since we can see that very specific initial conditions are required to reach an "unstable" critical point and thus perform many iterates with the macro algorithm. Hence we can pretend that, in general, Algorithm 1 will converge in a few number of iterates.

We will now consider examples in higher dimension to compare performances of the IHT algorithm with the proposed macro algorithm. The comparison will be done in terms of minimization of the ob- 
jective function $\mathrm{G}_{\ell_{0}}$. In other words we will say that an algorithm found a "better" (local) minimizer $\hat{\mathrm{x}}$ if it leads to a lower function value. To this end, we will not consider very high dimensional problems as the ones usually encountered in image processing but smaller ones (e.g. $M=7$ and $N=15)$ since, in this case, we are able to do a combinatorial search (as in $\S 4.2$ ) in a reasonable amount of time to find a global minimizer $\mathrm{x}^{\star}$ and the associated energy value $\mathrm{G}_{\ell_{0}}\left(\mathrm{x}^{\star}\right)$. Then we can compute the error $\left|\mathrm{G}_{\ell_{0}}(\hat{\mathrm{x}})-\mathrm{G}_{\ell_{0}}\left(\mathrm{x}^{\star}\right)\right|$ as a measure of performance. The lower the error the better the estimated solution $\hat{\mathrm{x}}$.

Figure 5 presents cumulative histograms of the errors $\left|\mathrm{G}_{\ell_{0}}(\hat{\mathrm{x}})-\mathrm{G}_{\ell_{0}}\left(\mathrm{x}^{\star}\right)\right|$ obtained from 1000 random generation of $A \in \mathbb{R}^{7 \times 15}$ and $d \in R^{7}$ from an uniform distribution. The IHT algorithm and the macro algorithm with IRl1 or FB are used with both an initialization with $A^{T} d$ or the solution of the $\ell_{1}$ relaxed problem. Different values of the hyper-parameter $\lambda$ are considered and the steps size of the IHT, FB and FISTA ${ }^{3}$ algorithms are fixed to $\frac{1}{L}$ where $L$ is the Lipschitz constant of the gradient of the data term in $\mathrm{G}_{\ell_{0}}$.

Such figures represent the proportion of the 1000 achievements where the algorithm have produced an error smaller than the value given on the $x$-axis. Then the $y$ value at zero corresponds to the proportion of the 1000 realizations where the algorithm reached a global optimum. The smallest $x$ for which the curve attains 1 provides the largest error obtained over all the generated problems. Therefore the faster the curve tends to 1 , the better the algorithm behaves.

We can see on Figure 5 that the macro algorithm always performs better than the IHT algorithm. This agrees with the fact that $\mathrm{G}_{\text {сЕц }}$ has less local minimizer than $\mathrm{G}_{\ell_{0}}$ which makes the macro algorithm more "robust" against local minimizers of $\mathrm{G}_{\ell_{0}}$. A slightly difference appears between the use of the IRl1 or FB as inner algorithm. It can illustrate a point outlined by the authors in [34] that is the ability of IRl1 to avoid local minimizers. The initialization has a huge effect on the solution obtained with the IHT algorithm. Initializing with the solution obtained by $\ell_{1}$ relaxation clearly improves the performance of the IHT algorithm. However this is not true for Algorithm 1 for $\lambda=0.5$ and $\lambda=1$ which is robust toward the initialization on these experiments. For the case $\lambda=0.1$ we also observe an improvement of the performance of the macro algorithm by initializing with the $\ell_{1}$ solution. One can note that the difference between IHT and macro algorithm is less important for low values of $\lambda$. This can be explained by the results presented in Table 1 where it was outlined that more strict local minimizer of $\mathrm{G}_{\ell_{0}}$ are removed with $\mathrm{G}_{\text {СЕLо }}$ when $\lambda$ is large. Results of Figure 5 show an interesting behaviour of the proposed macro algorithm which can be a good alternative to the IHT for the minimization of $\mathrm{G}_{\ell_{0}}$.

\section{Conclusions and perspectives}

In this paper we introduced and analyzed the CEL0 penalty which constitutes a continuous alternative to the $\ell_{0}$-norm in problem (1.4). The definition of this penalty follows from the study of the one dimensional case and the case of orthogonal matrices. For these special cases, we showed ( $\$ 2$ and $\S 3$ ) that the objective function $\mathrm{G}_{\mathrm{CEL} O}$, composed of a quadratic data term and the CEL0 penalty, is the convex hull of the functional, $\mathrm{G}_{\ell_{0}}$, of the initial $\ell_{0}$ penalized least squares problem (1.4). Then we studied the links between the minimizers of $\mathrm{G}_{\text {CЕLо }}$ and $\mathrm{G}_{\ell_{0}}$ for any $A \in \mathbb{R}^{M \times N}, d \in \mathbb{R}^{M}$ and $\lambda>0$. In this context, we proved two main results which are that global minimizers of $\mathrm{G}_{\text {CELo }}$ contain those of $\mathrm{G}_{\ell_{0}}$ and that a global minimizer of $\mathrm{G}_{\ell_{0}}$ can be easily obtained from each global minimizer of $\mathrm{G}_{\mathrm{CEL}}$ (Theorem 4.5). This last claim holds also for local minimizers of $\mathrm{G}_{\mathrm{CEL}}$ (Theorem 4.8). Moreover, we showed that the reciprocal of Theorem 4.8 is false and numerical experiments on low dimensional examples revealed that a large number of strict local minimizers of $\mathrm{G}_{\ell_{0}}$ are not critical points of $\mathrm{G}_{\text {сгцо }}$.

\footnotetext{
${ }^{3}$ FISTA [2] is used for the inner loop of IRl1 algorithm.
} 


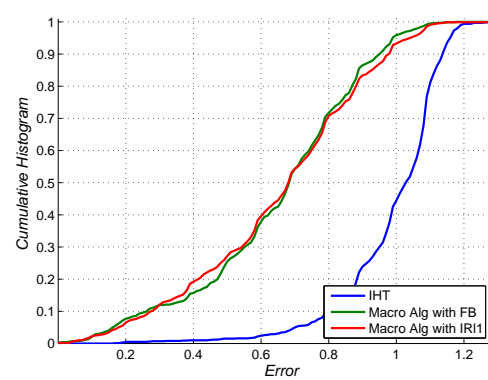

(a) Init $A^{T} d, \lambda=0.1$

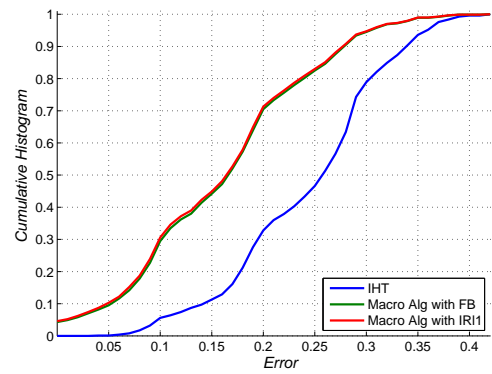

(d) Init $\ell_{1}, \lambda=0.1$

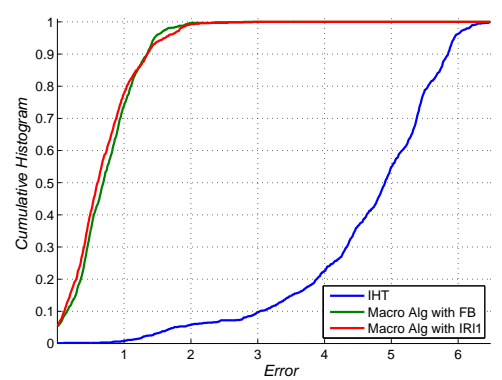

(b) Init $A^{T} d, \lambda=0.5$

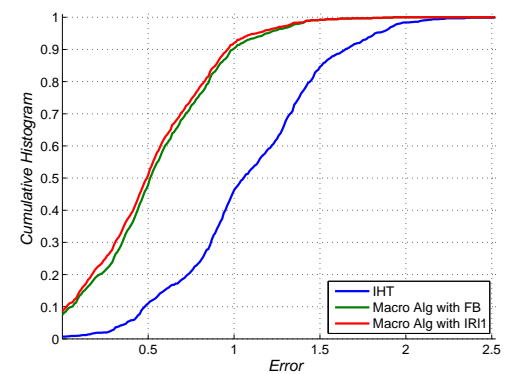

(e) Init $\ell_{1}, \lambda=0.5$

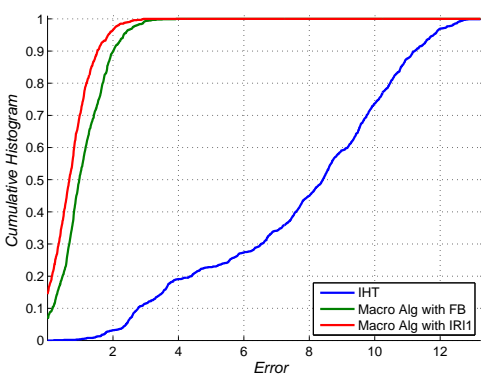

(c) Init $A^{T} d, \lambda=1$

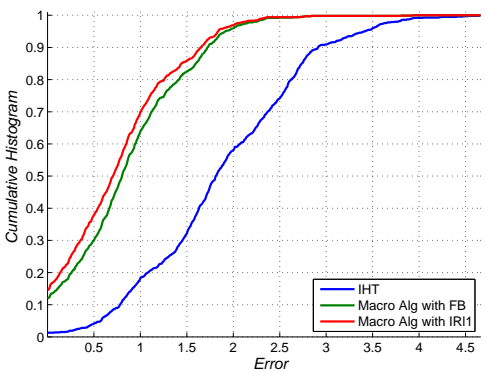

(f) Init $\ell_{1}, \lambda=1$

Figure 5: Cumulative histograms of the error $\left|\mathrm{G}_{\ell_{0}}(\hat{\mathrm{x}})-\mathrm{G}_{\ell_{0}}\left(\mathrm{x}^{\star}\right)\right|$ where $\hat{\mathrm{x}}$ is the estimated solution and $\mathrm{x}^{\star}$ is a global minimizer of $\mathrm{G}_{\ell_{0}}$. The histograms are computed from 1000 random matrices $A \in \mathbb{R}^{7 \times 15}$ and $d \in R^{7}$ generated from an uniform distribution. Two different initializations are considered: $\mathrm{x}^{0}=A^{T} d\left(\right.$ Init $\left.A^{T} d\right)$ and $\mathrm{x}^{0}=\mathrm{x}_{\ell_{1}}$ (Init $\ell_{1}$ ) the solution of the $\ell_{1}$ relaxed problem. The experiment is repeated for three values of $\lambda(0.1,0.5$ and 1$)$. For each configuration the estimation is performed using the IHT algorithm (blue) and the macro algorithm combined with IRl1 (red) or FB (green). The random seed is fixed at the same value for the six configurations in order to generate the same sequence of problems.

Furthermore, on these experiments, these points are the ones providing the higher values of $\mathrm{G}_{\ell_{0}}$ among all strict local minimizers of $\mathrm{G}_{\ell_{0}}$.

The analysis provided in $\S 4$ shows that it is preferable to address the $\ell_{0}$ penalized least squares problem (1.4) by minimizing $\mathrm{G}_{\mathrm{CEL} \text { o }}$ instead of $\mathrm{G}_{\ell_{0}}$. Moreover, $\mathrm{G}_{\mathrm{CELO}}$ has the nice property to be continuous unlike $\mathrm{G}_{\ell_{0}}$ and then nonsmooth nonconvex algorithms can be used to minimize $\mathrm{G}_{\text {CELo }}$ and thus $\mathrm{G}_{\ell_{0}}$.

In the context of such algorithms, we defined in $\S 5$ a macro algorithm for which we proved the convergence of the generated sequence to a (local) minimizer of $\mathrm{G}_{\ell_{0}}$ (Theorem 5.1). From any state of the art algorithm verifying a sufficient decrease condition and the convergence to a critical point of $\mathrm{G}_{\mathrm{CEL}}$, the proposed macro algorithm add an outer loop ensuring the convergence to a point that is both a critical point of $\mathrm{G}_{\text {CELO }}$ and a (local) minimizer of $\mathrm{G}_{\ell_{0}}$. Numerical illustrations showed an interesting behaviour of this macro algorithm in terms of $\mathrm{G}_{\ell_{0}}$ minimization.

As perspectives, it seems interesting to further investigate several theoretical and practical issues concerning the CEL0 penalty and associated algorithms.

- Lemma 4.1 provides a characterization of critical points of $\mathrm{G}_{\text {CELO }}$. What about a characterization of (local) minimizers of $\mathrm{G}_{\text {CELO }}$ ? As outlined previously, being a (local) minimizer of $\mathrm{G}_{\text {CELo }}$ is a necessary condition to be a global optimum of $\mathrm{G}_{\ell_{0}}$ which makes this question relevant. 
- Is there a similar way to define a penalty with the same properties as $\Phi_{\text {свьо when we consider }}$ $\|D \mathrm{x}\|_{0}$ where $D$ is a linear operator? This formulation, often encountered in image processing applications, need to be investigated.

- How can we define a smart rule for the selection step of the macro algorithm (line 7 of Algorithm 1)?

Finally, this work provides a new way to address the $\ell_{0}$ regularized least squares problem (1.4). Then one may focus on the development of new algorithms (or the improvement of existing ones) dedicated to the minimization of $\mathrm{G}_{\text {CELo }}$ which, unlike $\mathrm{G}_{\ell_{0}}$, has the nice property to be continuous and convex with respect to each variable.

\section{A The 1D biconjugate of $g$ (eq. 2.1)}

Let us first determine the expression (2.4) of the conjugate of $\mathrm{g}$ which is the solution of the problem (2.3). In the following we will denote by $h_{u^{\star}}$ the objective function of (2.3) (with $f=\mathrm{g}$ ) that is

$$
h_{u^{\star}}(u)=u^{\star} u-\mathrm{g}(u)=u^{\star} u-\frac{1}{2}(a u-d)^{2}-\lambda|u|_{0} .
$$

From the definition of $|u|_{0}$ in (2.2), it is clear that $h_{u^{\star}}$ has two local maxima on $\mathbb{R}$. The first one is reached for $u_{0}=0$ and corresponds to

$$
h_{u^{\star}}\left(u_{0}\right)=-\frac{d^{2}}{2} .
$$

The second one, reached at $u_{1} \neq 0$, corresponds to the continuous part of $h_{u^{\star}}$ (i.e. when $|u|_{0}=1$ ). Then $u_{1}$ solves

$$
h_{u^{\star}}^{\prime}\left(u_{1}\right)=0, u_{1} \neq 0 \Longleftrightarrow u^{\star}-a^{2} u_{1}+a d=0, u_{1} \neq 0,
$$

which is equivalent to

$$
u_{1}=\frac{1}{a^{2}}\left(u^{\star}+a d\right), u^{\star} \neq-a d,
$$

and we have

$$
h_{u^{\star}}\left(u_{1}\right)=\frac{\left(u^{\star}\right)^{2}}{2 a^{2}}+\frac{u^{\star} d}{a}-\lambda=\frac{1}{2 a^{2}}\left(u^{\star}+a d\right)^{2}-\frac{d^{2}}{2}-\lambda, u^{\star} \neq-a d .
$$

If $u^{\star}=-a d$ then $u_{0}=u_{1}=0$ and the associated value of $h_{u^{\star}}$ is given by (A.2). Otherwise we have to compare the values given in (A.2) and (A.5) to know if $u_{0}$ or $u_{1}$ is global maximizer,

$$
\begin{aligned}
& \frac{1}{2 a^{2}}\left(u^{\star}+a d\right)^{2}-\frac{d^{2}}{2}-\lambda+\frac{d^{2}}{2} \geq 0 \\
\Longleftrightarrow & \left|u^{\star}+a d\right| \geq \sqrt{2 \lambda} a
\end{aligned}
$$

Finally, the conjugate $\mathrm{g}^{\star}$ is given by,

$$
\mathrm{g}^{\star}\left(u^{\star}\right)=\left\{\frac{1}{2 a^{2}}\left(u^{\star}+a d\right)^{2}-\lambda\right\} \mathbb{1}_{\left\{\left|u^{\star}+a d\right| \geq \sqrt{2 \lambda} a\right\}}-\frac{d^{2}}{2} .
$$

We can now calculate the biconjugate of $g$ which is given by

$$
\mathrm{g}^{\star \star}(u)=\sup _{v \in \mathbb{R}} u v-\mathrm{g}^{\star}(v) .
$$


Again we will denote by $h_{u}^{\star}$ the objective function of (A.7),

$$
h_{u}^{\star}(v)=u v-\mathrm{g}^{\star}(v)=u v-\left\{\frac{1}{2 a^{2}}(v+a d)^{2}-\lambda\right\} \mathbb{1}_{\{|v+a d| \geq \sqrt{2 \lambda} a\}}+\frac{d^{2}}{2} .
$$

We have to consider two cases:

1. if $|v+a d|<\sqrt{2 \lambda} a$,

$$
h_{u}^{\star}(v)=u v+\frac{d^{2}}{2} .
$$

Since $h_{u}^{\star}$ is linear and that we want to maximize it on $[-a d-\sqrt{2 \lambda} a,-a d+\sqrt{2 \lambda} a]$, the entire interval is solution if $u=0$ and otherwise the maximizer $v_{1}$ is one of the bounds of this interval depending on the sign of $u$. Then, $v_{1}$ can be written as

$$
v_{1}=-a d+\operatorname{sign}(u) \sqrt{2 \lambda} a,
$$

and we have

$$
h_{u}^{\star}\left(v_{1}\right)=-a d u+\operatorname{sign}(u) u \sqrt{2 \lambda} a+\frac{d^{2}}{2}=-a d u+|u| \sqrt{2 \lambda} a+\frac{d^{2}}{2} .
$$

2. if $|v+a d| \geq \sqrt{2 \lambda} a$,

$$
h_{u}^{\star}(v)=u v-\frac{1}{2 a^{2}}(v+a d)^{2}+\lambda+\frac{d^{2}}{2},
$$

and the maximizer $v_{2}$ solves

$$
\left(h_{u}^{\star}\right)^{\prime}\left(v_{2}\right)=0 \Longleftrightarrow u-\frac{1}{a^{2}}\left(v_{2}+a d\right)=0,
$$

which is equivalent to

$$
v_{2}=a^{2} u-a d .
$$

Then we must insure that

$$
\left|v_{2}+a d\right| \geq \sqrt{2 \lambda} a \Longleftrightarrow|u| \geq \frac{\sqrt{2 \lambda}}{a} .
$$

Finally, if $u$ verifies the condition (A.15) then $v_{2}$ given by (A.14) is the maximizer of $h_{u}^{\star}$ on $\mathbb{R} \backslash[-a d-\sqrt{2 \lambda} a,-a d+\sqrt{2 \lambda} a]$ and

$$
h_{u}^{\star}\left(v_{2}\right)=\frac{a^{2} u^{2}}{2}-a u d+\frac{d^{2}}{2}+\lambda=\frac{1}{2}(a u-d)^{2}+\lambda .
$$

Otherwise the supremum of $h_{u}^{\star}$ on $\mathbb{R} \backslash[-a d-\sqrt{2 \lambda} a,-a d+\sqrt{2 \lambda} a]$ is given by (A.11).

From the two previous cases and noticing that (A.11) and (A.16) yield the same value at $|u|=\frac{\sqrt{2 \lambda}}{a}$, it is straightforward that

$$
\mathrm{g}^{\star \star}(u)= \begin{cases}-a d u+|u| \sqrt{2 \lambda} a+\frac{d^{2}}{2} & \text { if }|u| \leq \frac{\sqrt{2 \lambda}}{a}, \\ \frac{1}{2}(a u-d)^{2}+\lambda & \text { if }|u| \geq \frac{\sqrt{2 \lambda}}{a}\end{cases}
$$




\section{B Generalized gradient and proof of Lemma 4.1 (Critical points of $\left.\mathrm{G}_{\mathrm{CELO}}\right)$}

\section{B.1 Generalized Gradient}

In this section we recall the definition and properties of the generalized gradient introduced by Clarke [11]. This generalized gradient extends the notion of subdifferential for convex functions to nonconvex ones.

Definition B.1 (Local Lipschitz condition). A function $f$ is said to be locally Lipschitz at $\mathrm{x}$ if,

$$
\exists \varepsilon>0, \forall\left(\mathrm{y}, \mathrm{y}^{\prime}\right) \in \mathcal{B}(\mathrm{x}, \varepsilon)^{2}\left|f(\mathrm{y})-f\left(\mathrm{y}^{\prime}\right)\right| \leq K\left\|\mathrm{y}-\mathrm{y}^{\prime}\right\|,
$$

where $K$ is a nonnegative scalar and $\mathcal{B}(\mathrm{x}, \varepsilon)$ is a $\varepsilon$-neighborhood of $\mathrm{x}$.

Definition B.2 (Generalized gradient (Clarke [11])). The generalized gradient of a locally Lipschitz function $f: \mathbb{R}^{N} \rightarrow \mathbb{R}$ at $\mathrm{x}$, denoted $\partial f(\mathrm{x})$, is defined by,

$$
\partial f(\mathrm{x}):=\left\{\xi \in \mathbb{R}^{N}: f^{0}(\mathrm{x}, v) \geq\langle v, \xi\rangle \forall v \in \mathbb{R}^{N}\right\},
$$

where $f^{0}(\mathrm{x}, v)$ denotes the generalized directional derivative of $f$ at $\mathrm{x}$ in the direction $v$,

$$
f^{0}(\mathrm{x}, v)=\limsup _{\substack{\mathrm{y} \rightarrow \mathrm{x} \\ \eta \downarrow 0}} \frac{f(\mathrm{y}+\eta v)-f(\mathrm{y})}{\eta} .
$$

When $f$ is smooth, $\partial f(\mathrm{x})$ reduces to the singleton $\{\nabla f(\mathrm{x})\}[11$, Proposition 2.2.4 and related Corollary] and, in the convex case, the Clarke generalized gradient coincides with the classical subdifferential of convex functions [11, Proposition 2.2.7].

Proposition B.1 (Local Extrema (Clarke [11])). If $f$ attains a local minimum or maximum at $\mathrm{x}$, then $0 \in \partial f(\mathrm{x})$.

The condition $0 \in \partial f(\mathrm{x})$ is thus necessary for $\mathrm{x}$ being a local minimizer or maximizer of $f$. We call critical points of $f$ the $\mathrm{x} \in \mathbb{R}^{N}$ verifying $0 \in \partial f(\mathrm{x})$.

From definition B.2 we can compute $\partial \phi$ where $\phi$ is defined by (2.9). Since for all $u \neq 0, \phi$ is differentiable, we have

$$
\forall u \neq 0, \partial \phi(a, \lambda ; u)= \begin{cases}\operatorname{sign}(u) \sqrt{2 \lambda} a-a^{2} u & \text { if } 0<|u| \leq \frac{\sqrt{2 \lambda}}{a} \\ 0 & \text { if }|u| \geq \frac{\sqrt{2 \lambda}}{a}\end{cases}
$$

The remaining case is $u=0$. Let us start with the computation of the generalized directional derivative (B.3) at $u=0$,

$$
\begin{aligned}
\forall v \in \mathbb{R}, f^{0}(0, v) & =\limsup _{\substack{\mathrm{y} \rightarrow 0 \\
\eta \downarrow 0}} \frac{\phi(\mathrm{y}+\eta v)-\phi(\mathrm{y})}{\eta} \\
& =\limsup _{\substack{\mathrm{y} \rightarrow 0 \\
\eta \downarrow 0}} \frac{1}{2 \eta}\left[(a|\mathrm{y}|-\sqrt{2 \lambda})^{2}-(a|\mathrm{y}+\eta v|-\sqrt{2 \lambda})^{2}\right] .
\end{aligned}
$$


Then one can see that,

$$
f^{0}(0, v)=a|v| \sqrt{2 \lambda}
$$

Therefore, from (B.2), $\partial \phi(0)$ contains all $\xi \in \mathbb{R}^{N}$ verifying $a|v| \sqrt{2 \lambda} \geq \xi v \forall v \in \mathbb{R}^{N}$ that is,

$$
\partial \phi(a, \lambda ; 0)=[-a \sqrt{2 \lambda}, a \sqrt{2 \lambda}] .
$$

\section{B.2 Proof of Lemma 4.1}

First of all, by hypothesis we have

$$
\forall i \in \mathbb{I}_{N},\left\|a_{i}\right\|>0
$$

and then equation (4.4) is well-defined. Since the data term in $\mathrm{G}_{\mathrm{CEL}}$ is differentiable, we have

$$
\forall \mathrm{x} \in \mathbb{R}^{N}, \partial \mathrm{G}_{\mathrm{CEL} O}(\mathrm{x})=A^{T}(A \mathrm{x}-d)+\partial \Phi_{\mathrm{CELO}}(\mathrm{x}) .
$$

Let $\hat{\mathrm{x}} \in \mathbb{R}^{N}$ be a critical point of $\mathrm{G}_{\mathrm{CEL} O}$, i.e.

$$
0_{\mathbb{R}^{N}} \in \partial \mathrm{G}_{\mathrm{CELO}}(\hat{\mathrm{x}})
$$

which, following (B.8), is equivalent to

$$
0_{\mathbb{R}^{N}} \in \prod_{i \in \mathbb{I}_{N}}\left[A^{T}(A \hat{\mathrm{x}}-d)\right]_{i}+\partial \phi\left(\left\|a_{i}\right\|, \lambda ; \hat{\mathrm{x}}_{i}\right) .
$$

Then from (B.4) and (B.6) the previous equation is equivalent to

$$
\begin{aligned}
& \forall i \in \mathbb{I}_{N}, \begin{cases}0 \in\left[\left\langle a_{i}, A \hat{\mathrm{x}}-d\right\rangle-\sqrt{2 \lambda}\left\|a_{i}\right\|,\left\langle a_{i}, A \hat{\mathrm{x}}-d\right\rangle+\sqrt{2 \lambda}\left\|a_{i}\right\|\right] & \text { iff } \hat{\mathrm{x}}_{i}=0, \\
0=\left\langle a_{i}, A \hat{\mathrm{x}}-d\right\rangle-\left\|a_{i}\right\|^{2} \hat{\mathrm{x}}_{i}+\operatorname{sign}\left(\hat{\mathrm{x}}_{i}\right) \sqrt{2 \lambda}\left\|a_{i}\right\| & \text { iff } 0<\left|\hat{\mathrm{x}}_{i}\right| \leq \frac{\sqrt{2 \lambda}}{\left\|a_{i}\right\|} \\
0=\left\langle a_{i}, A \hat{\mathrm{x}}-d\right\rangle & \text { iff }\left|\hat{\mathrm{x}}_{i}\right| \geq \frac{\sqrt{2 \lambda}}{\left\|a_{i}\right\|},\end{cases} \\
& \Longleftrightarrow \forall i \in \mathbb{I}_{N}, \begin{cases}\left|\left\langle a_{i}, A \hat{\mathrm{x}}^{(i)}-d\right\rangle\right| \leq \sqrt{2 \lambda}\left\|a_{i}\right\| & \text { iff } \hat{\mathrm{x}}_{i}=0, \\
0=\left\langle a_{i}, A \hat{\mathrm{x}}^{(i)}-d\right\rangle+\operatorname{sign}\left(\hat{\mathrm{x}}_{i}\right) \sqrt{2 \lambda}\left\|a_{i}\right\| & \text { iff } 0<\left|\hat{\mathrm{x}}_{i}\right| \leq \frac{\sqrt{2 \lambda}}{\left\|a_{i}\right\|}, \\
0=\left\langle a_{i}, A \hat{\mathrm{x}}^{(i)}-d\right\rangle+\left\|a_{i}\right\|^{2} \hat{\mathrm{x}}_{i} & \text { iff }\left|\hat{\mathrm{x}}_{i}\right| \geq \frac{\sqrt{2 \lambda}}{\left\|a_{i}\right\|},\end{cases} \\
& \Longleftrightarrow \forall i \in \mathbb{I}_{N}, \begin{cases}\hat{\mathrm{x}}_{i}=0 & \text { iff }\left|\left\langle a_{i}, A \hat{\mathrm{x}}^{(i)}-d\right\rangle\right| \leq \sqrt{2 \lambda}\left\|a_{i}\right\|, \\
\hat{\mathrm{x}}_{i}=-s_{i} t, t \in\left[0, \frac{\sqrt{2 \lambda}}{\left\|a_{i}\right\|}\right] & \text { iff }\left|\left\langle a_{i}, A \hat{\mathrm{x}}^{(i)}-d\right\rangle\right|=\sqrt{2 \lambda}\left\|a_{i}\right\| \\
\hat{\mathrm{x}}_{i}=-\frac{\left\langle a_{i}, A \hat{\mathrm{x}}^{(i)}-d\right\rangle}{\left\|a_{i}\right\|^{2}} & \text { iff }\left|\left\langle a_{i}, A \hat{\mathrm{x}}^{(i)}-d\right\rangle\right| \geq \sqrt{2 \lambda}\left\|a_{i}\right\|\end{cases}
\end{aligned}
$$

where $\hat{\mathrm{x}}^{(i)}=\left(\hat{\mathrm{x}}_{1}, \cdots, \hat{\mathrm{x}}_{i-1}, 0, \hat{\mathrm{x}}_{i+1}, \cdots, \hat{\mathrm{x}}_{N}\right)$ and $s_{i}=\operatorname{sign}\left(\left\langle a_{i}, A \hat{\mathrm{x}}^{(i)}-d\right\rangle\right)$. This completes the proof.

\section{Proof of Theorem 4.5 (Link between global minimizers of $\mathrm{G}_{\ell_{0}}$ and $\left.\mathrm{G}_{\mathrm{CELO}}\right)$}

Let first notice that by definition of $\mathrm{G}_{\mathrm{CEL}}$ in $(4.1)$, we have,

$$
\mathrm{G}_{\mathrm{CEL} 0}(\mathrm{x})=\mathrm{G}_{\ell_{0}}(\mathrm{x}), \forall \mathrm{x} \in \mathbb{R}^{N} \backslash S \text { where } S:=\left\{\mathrm{x} \in \mathbb{R}^{N}: \sigma^{-}(\mathrm{x}) \neq \emptyset\right\} .
$$


(i) Let $\hat{\mathrm{x}} \in \mathbb{R}^{N}$ be a global minimizer of $\mathrm{G}_{\ell_{0}}$ (there exists at least one from [32, Theorem 4.4]). By Proposition 4.2 and with (C.1) we have,

$$
\mathrm{G}_{\mathrm{CEL} O}(\hat{\mathrm{x}})=\mathrm{G}_{\ell_{0}}(\hat{\mathrm{x}}) .
$$

Suppose that there exists $\overline{\mathrm{x}} \in \mathbb{R}^{N}$ such that,

$$
\mathrm{G}_{\mathrm{CELO}}(\overline{\mathrm{x}})<\mathrm{G}_{\mathrm{CELO}}(\hat{\mathrm{x}})=\mathrm{G}_{\ell_{0}}(\hat{\mathrm{x}}) .
$$

- if $\overline{\mathrm{x}} \in \mathbb{R}^{N} \backslash S$ then from (C.1),

$$
\mathrm{G}_{\ell_{0}}(\overline{\mathrm{x}})=\mathrm{G}_{\mathrm{CEL}}(\overline{\mathrm{x}})<\mathrm{G}_{\mathrm{CEL} 0}(\hat{\mathrm{x}})=\mathrm{G}_{\ell_{0}}(\hat{\mathrm{x}}),
$$

which contradicts the fact that $\hat{\mathrm{x}}$ is a global minimizer of $\mathrm{G}_{\ell_{0}}$.

- if $\overline{\mathrm{x}} \in S$, then let $i \in \sigma^{-}(\overline{\mathrm{x}})$ and $\mathrm{G}_{\mathrm{CELO}}^{i}$ be the restriction of $\mathrm{G}_{\mathrm{CELO}}$ to the ith variable at $\overline{\mathrm{x}}$. It is easy to show (see the proof of Proposition 4.10) that,

$$
\forall t \in \mathbb{R}, \mathrm{G}_{\mathrm{CELO}}^{i}(t)=\mathrm{G}_{\mathrm{CELO}}\left(\overline{\mathrm{x}}^{(i)}+t e_{i}\right)=\frac{1}{2}\left(\left\|a_{i}\right\| t-\tilde{d}\right)^{2}+\phi\left(\left\|a_{i}\right\|, \lambda ; t\right)+C,
$$

where $C$ is a constant independent of $t$ and $\tilde{d}=\left\langle a_{i}, d-A \overline{\mathrm{x}}^{(i)}\right\rangle /\left\|a_{i}\right\| \in \mathbb{R}$. Let

$$
\hat{t} \in \underset{t \in \mathbb{R}}{\arg \min } \mathrm{G}_{\mathrm{cEL}}^{i}(t),
$$

then such a $\hat{t}$ verifies,

$$
\hat{t}=\left\{\begin{array}{ll}
0 & \text { if }|\tilde{d}| \leq \sqrt{2 \lambda} \\
\operatorname{sign}(\tilde{d}) t, t \in\left[0, \frac{\tilde{d}}{\left\|a_{i}\right\|}\right] & \text { if }|\tilde{d}|=\sqrt{2 \lambda} \\
\frac{\tilde{d}}{\left\|a_{i}\right\|} & \text { if }|\tilde{d}| \geq \sqrt{2 \lambda}
\end{array},\right.
$$

Let define

$$
\overline{\mathrm{x}}^{1}=\overline{\mathrm{x}}^{(i)}+e_{i} \frac{\tilde{d}}{\left\|a_{i}\right\|} \mathbb{1}_{\{|\tilde{d}|>\sqrt{2 \lambda}\}} .
$$

Clearly, $\left|\overline{\mathrm{x}}_{i}^{1}\right| \in\left\{0, \tilde{d} /\left\|a_{i}\right\|\right\}$ and from (C.6) and (C.7) $\mathrm{G}_{\mathrm{CELO}}\left(\overline{\mathrm{x}}^{1}\right) \leq \mathrm{G}_{\mathrm{CELO}}(\overline{\mathrm{x}})$. Moreover $\# \sigma^{-}\left(\overline{\mathrm{x}}^{1}\right)=$ $\# \sigma^{-}(\overline{\mathrm{x}})-1$. This process can then be repeated for another $i \in \sigma^{-}\left(\overline{\mathrm{x}}^{1}\right) \subset \sigma^{-}(\overline{\mathrm{x}})$ and one can construct a sequence $\left(\overline{\mathrm{x}}^{k}\right)_{k \in\{1 \cdots K\}}$ (where $\left.K=\# \sigma^{-}(\overline{\mathrm{x}})\right)$ such that,

$$
\mathrm{G}_{\mathrm{CELO}}\left(\overline{\mathrm{x}}^{K}\right) \leq \mathrm{G}_{\mathrm{CELO}}\left(\overline{\mathrm{x}}^{K-1}\right) \leq \cdots \leq \mathrm{G}_{\mathrm{CEED}}\left(\overline{\mathrm{x}}^{1}\right) \leq \mathrm{G}_{\mathrm{CEEO}}(\overline{\mathrm{x}}),
$$

and,

$$
\# \sigma^{-}\left(\overline{\mathrm{x}}^{K}\right)=\# \sigma^{-}\left(\overline{\mathrm{x}}^{K-1}\right)-1=\cdots=\# \sigma^{-}(\overline{\mathrm{x}})-\# \sigma^{-}(\overline{\mathrm{x}})=0 .
$$

Then from (C.1), (C.3), (C.9) and (C.10) we get,

$$
\mathrm{G}_{\ell_{0}}\left(\overline{\mathrm{x}}^{K}\right)=\mathrm{G}_{\mathrm{CELO}}\left(\overline{\mathrm{x}}^{K}\right) \leq \mathrm{G}_{\mathrm{CELD}}(\overline{\mathrm{x}})<\mathrm{G}_{\mathrm{CELO}}(\hat{\mathrm{x}})=\mathrm{G}_{\ell_{0}}(\hat{\mathrm{x}}),
$$

which contradicts the fact that $\hat{\mathrm{x}}$ is a global minimizer of $\mathrm{G}_{\ell_{0}}$. Hence, $\nexists \overline{\mathrm{x}} \in \mathbb{R}^{N}$ such that $\mathrm{G}_{\mathrm{CELO}}(\overline{\mathrm{x}})<\mathrm{G}_{\mathrm{CEL} O}(\hat{\mathrm{x}})$ and then $\hat{\mathrm{x}}$ is a global minimizer of $\mathrm{G}_{\mathrm{CEL} 0}$.

(ii) Let $\hat{\mathrm{x}} \in \mathbb{R}^{N}$ be a global minimizer of $\mathrm{G}_{\mathrm{CEL}}$. By Lemma 4.4 , $\hat{\mathrm{x}}^{0}$, defined by (4.13), is also a global minimizer of $\mathrm{G}_{\text {CEL }}$ such that $\sigma^{-}\left(\hat{\mathrm{x}}^{0}\right)=\emptyset$ and, with (C.1), we get

$$
\mathrm{G}_{\mathrm{CELO}}(\hat{\mathrm{x}})=\mathrm{G}_{\text {CELo }}\left(\hat{\mathrm{x}}^{0}\right)=\mathrm{G}_{\ell_{0}}\left(\hat{\mathrm{x}}^{0}\right) \text {. }
$$

Since $\mathrm{G}_{\text {СЕо }}$ underestimate $\mathrm{G}_{\ell_{0}}$ (see Remark 4.1), $\hat{\mathrm{x}}^{0}$ is a global minimizer of $\mathrm{G}_{\ell_{0}}$. 


\section{Proof of Theorem 5.1 (Convergence of the macro algorithm)}

In this proof we consider the restriction of $\mathrm{G}_{\text {CELo }}$ defined by,

$$
\mathrm{G}_{\mathrm{CELO}}^{\mathcal{E}}: \mathcal{E} \longrightarrow \mathbb{R}
$$

where $\mathcal{E}:=\left\{\mathrm{x} \in \mathbb{R}^{N}, \mathrm{G}_{\text {CELO }}(\mathrm{x})<\mathrm{G}_{\text {CELO }}\left(\mathrm{x}^{\text {init }}\right)\right\} \subset \mathbb{R}^{N}$. Note that since the macro algorithm is a descent algorithm it works only on the restriction $\mathrm{G}_{\text {CELO }}^{\mathcal{E}}$ and then we can limit the proof to this restriction.

Let us denote $\mathcal{C}$ the set of Clarke critical points of $\mathrm{G}_{\mathrm{CELO}}^{\mathcal{E}}$. We start the proof by showing that the image of $\mathcal{C}$ by $\mathrm{G}_{\text {CELO }}^{\mathcal{E}}$, denoted $\mathrm{G}_{\text {CELO }}^{\mathcal{E}}(\mathcal{C})$, is finite. Let $\hat{\mathrm{x}} \in \mathcal{C}, \hat{\sigma}=\sigma(\hat{\mathrm{x}})$ and $\hat{\sigma}^{-}=\sigma^{-}(\hat{\mathrm{x}})$. Then from the first accolade in the proof of Lemma 4.1 (Appendix B.2), $\hat{\mathrm{x}}$ solves

$$
\begin{aligned}
\forall i \in \hat{\sigma} \begin{cases}0=\left\langle a_{i}, A \hat{\mathrm{x}}-d\right\rangle-\left\|a_{i}\right\|^{2} \hat{\mathrm{x}}_{i}+\operatorname{sign}\left(\hat{\mathrm{x}}_{i}\right) \sqrt{2 \lambda}\left\|a_{i}\right\| & \text { iff } i \in \hat{\sigma}^{-}, \\
0=\left\langle a_{i}, A \hat{\mathrm{x}}-d\right\rangle & \text { iff } i \in \hat{\sigma} \backslash \hat{\sigma}^{-} .\end{cases} \\
\qquad\left\{\begin{array}{l}
0=\left(A_{\hat{\sigma}^{-}}\right)^{T}\left(A_{\hat{\sigma}} \hat{\mathrm{x}}_{\hat{\sigma}}-d\right)-D_{\hat{\sigma}^{-}} \hat{\mathrm{x}}_{\hat{\sigma}^{-}}+\sqrt{2 \lambda} b_{\hat{\sigma}^{-}}, \\
0=\left(A_{\hat{\sigma} \backslash \hat{\sigma}^{-}}\right)^{T}\left(A_{\hat{\sigma}} \hat{\mathrm{x}}_{\hat{\sigma}}-d\right) .
\end{array}\right.
\end{aligned}
$$

where $D \in \mathbb{R}^{N \times N}$ is a diagonal matrix whose entries are given by $D_{i i}=\left\|a_{i}\right\|^{2} \mathbb{1}_{\left\{i \in \hat{\sigma}^{-}\right\}}$and $b \in \mathbb{R}^{N}$ such that $b_{i}=\operatorname{sign}\left(\hat{\mathrm{x}}_{i}\right)\left\|a_{i}\right\| \mathbb{1}_{\left\{i \in \hat{\sigma}^{-}\right\}}$. Then (D.3) can be written as

$$
\left[\left(A_{\hat{\sigma}}\right)^{T} A_{\hat{\sigma}}-D_{\hat{\sigma}}\right] \hat{\mathrm{x}}_{\hat{\sigma}}=\left(A_{\hat{\sigma}}\right)^{T} d-\sqrt{2 \lambda} b_{\hat{\sigma}} .
$$

Therefore, belonging to $\mathcal{E}$ and solving (D.4) is a necessary ${ }^{4}$ condition to be a critical point of $\mathrm{G}_{\mathrm{CEL} O}$. $^{\mathcal{E}}$. Let us now take $\omega \subset \mathbb{I}_{N}, \omega^{-} \subset \omega$ and let $\overline{\mathrm{x}} \in \mathbb{R}^{N}$ solve

$$
\left[\left(A_{\omega}\right)^{T} A_{\omega}-D_{\omega}\right] \overline{\mathrm{x}}_{\omega}=\left(A_{\omega}\right)^{T} d-\sqrt{2 \lambda} b_{\omega}
$$

where $b_{i}= \pm\left\|a_{i}\right\| \mathbb{1}_{\left\{i \in \omega^{-}\right\}}$and $D_{i i}=\left\|a_{i}\right\|^{2} \mathbb{1}_{\left\{i \in \omega^{-}\right\}}$. Then such a $\overline{\mathrm{x}}$ is a critical point of $\mathrm{G}_{\text {CELo }}^{\mathcal{E}}$ if and only if $\overline{\mathrm{x}} \in \mathcal{E}$, solves (D.4) (i.e. $\sigma(\overline{\mathrm{x}})=\omega, \sigma^{-}(\overline{\mathrm{x}})=\omega^{-}$and $\operatorname{sign}\left(\overline{\mathrm{x}}_{i}\right)=\operatorname{sign}\left(b_{i}\right), \forall i \in \omega^{-}$) and verifies $\left|\left\langle a_{i}, A \overline{\mathrm{x}}^{(i)}-d\right\rangle\right| \leq \sqrt{2 \lambda}\left\|a_{i}\right\|, \forall i \in \sigma(\overline{\mathrm{x}})^{c}$. A finite number of systems like (D.5) can be constructed by choosing, $\omega, \omega^{-}$and the sign of the nonzero entries of $b$. Indeed the number of subset $\omega \subset \mathbb{I}_{N}$ is equal to $\sum_{k=1}^{N}\left(\begin{array}{l}N \\ k\end{array}\right)$ and similarly the number of subset $\omega^{-} \subset \omega$ is equal to $\sum_{l=1}^{\# \omega}\left(\begin{array}{c}\# \omega \\ l\end{array}\right)$. Then we have the choice of the sign for the nonzero entries of $b$ that is $2^{\# \omega^{-}}$possibilities. Finally one can generate

$$
\sum_{k=1}^{N}\left(\begin{array}{l}
N \\
k
\end{array}\right) \times \sum_{l=1}^{k}\left(\begin{array}{l}
k \\
l
\end{array}\right) \times\left(2^{l}\right),
$$

different systems. This number is huge but finite. We recall that solutions, $\overline{\mathrm{x}}$, of these systems are critical points of $\mathrm{G}_{\text {CELO }}^{\mathcal{E}}$ if and only if they belong to $\mathcal{E}$, solve (D.4) and verifies $\left|\left\langle a_{i}, A \overline{\mathrm{x}}^{(i)}-d\right\rangle\right| \leq$ $\sqrt{2 \lambda}\left\|a_{i}\right\|, \forall i \in \sigma(\overline{\mathrm{x}})^{c}$. Thus the number of systems like (D.5) leading to critical points of $\mathrm{G}_{\mathrm{CEL}}^{\mathcal{E}}$ is smaller than (D.6).

Systems like (D.5) can admit a unique solution if $\operatorname{rank}\left(\left(A_{\omega}\right)^{T} A_{\omega}-D_{\omega}\right)=\# \omega$ or, either none or an infinity of solutions if $\operatorname{rank}\left(\left(A_{\omega}\right)^{T} A_{\omega}-D_{\omega}\right) \leq \# \omega-1$. When such a system admits an infinity of solutions, these solutions define a vector subspace of $\mathbb{R}^{N}$. Among them, the ones which are critical points of $\mathrm{G}_{\text {cEL }}^{\mathcal{E}}$ belong to the intersection of this vector subspace with $\mathcal{E}$ and $E$ defined by,

$$
E:=I_{0} \cap \prod_{i \in \mathbb{I}_{N}} I_{i}
$$

\footnotetext{
${ }^{4}$ but not sufficient since from Lemma 4.1, a critical point of $\mathrm{G}_{\text {CELo }}$ also has to verify $\left|\left\langle a_{i}, A \hat{\mathrm{x}}^{(i)}-d\right\rangle\right| \leq \sqrt{2 \lambda}\left\|a_{i}\right\|, \forall i \in \hat{\sigma}^{c}$, condition which is not included in (D.4).
} 
where,

$$
I_{i}= \begin{cases}\{0\} & \text { if } i \in \omega^{c}, \\ {\left[-\frac{\sqrt{2 \lambda}}{\left\|a_{i}\right\|}, 0[,\right.} & \text { if } i \in \omega^{-} \text {and } b_{i}<0, \\ \left.0, \frac{\sqrt{2 \lambda}}{\left\|a_{i}\right\|}\right], & \text { if } i \in \omega^{-} \text {and } b_{i}>0, \\ \left.-\infty,-\frac{\sqrt{2 \lambda}}{\left\|a_{i}\right\|}\right] \cup\left[\frac{\sqrt{2 \lambda}}{\left\|a_{i}\right\|},+\infty[\right. & \text { if } i \in \omega \backslash \omega^{-},\end{cases}
$$

and

$$
I_{0}=\bigcap_{i \in \omega^{c}}\left\{\mathrm{x} \in \mathbb{R}^{N}:\left|\left\langle a_{i}, A \mathrm{x}^{(i)}-d\right\rangle\right| \leq \sqrt{2 \lambda}\left\|a_{i}\right\|\right\},
$$

One can see that E (eq. D.7) is composed of a finite number of connected components in $\mathbb{R}^{N}$. Indeed, since $\mathrm{x} \mapsto\left\langle a_{i}, A \mathrm{x}^{(i)}-d\right\rangle$ is linear, each set of the intersection defining $I_{0}$ is a connected component in $\mathbb{R}^{N}$ and therefore $I_{0}$ is also a connected component in $\mathbb{R}^{N}$. Finally, intersecting $I_{0}$ with the Cartesian product of the $I_{i}\left(i \in \mathbb{I}_{N}\right)$ proves the above affirmation.

Then one system like (D.5) results at most in a finite number of connected component in $\mathcal{C}$. Combining that with (D.6) we get that $\mathcal{C}$ contains a finite number of connected components. Following [1],

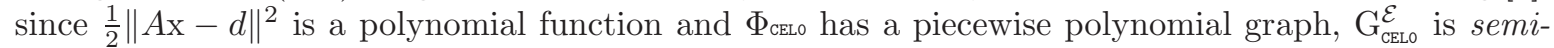
algebraic and then subanalytic. Hence we have all the requirements of [5, Theorem 7] which states that $\mathrm{G}_{\text {cELo }}^{\mathcal{E}}$ is constant on each connected component of $\mathcal{C}$. Consequently $\mathrm{G}_{\text {cELo }}^{\mathcal{E}}(\mathcal{C})$ is a finite set.

From $\mathrm{H} 1$ and by construction of the algorithm, $\forall k \in \mathbb{N}, \mathrm{x}^{k} \in \mathcal{C}$. Then, following the same arguments as in the proof of Lemma 4.4, setting one $\mathrm{x}_{i}^{k}$ such that $i \in \sigma_{k}^{-}$to zero does not change the value of $\mathrm{G}_{\text {cELO }}^{\mathcal{E}}$. Hence $\mathrm{G}_{\text {cELo }}^{\mathcal{E}}\left(\mathrm{x}^{t e m p}\right)=\mathrm{G}_{\text {cELO }}^{\mathcal{E}}\left(\mathrm{x}^{k}\right)$. With hypothesis $\mathrm{H} 1$ and $\mathrm{H} 2$ on Alg we get

$$
\forall k \in \mathbb{N}, \quad \text { and } \mathrm{G}_{\text {cELo }}^{\mathcal{E}}\left(\mathrm{x}^{k+1}\right) \leq \mathrm{G}_{\text {cELo }}^{\mathcal{E}}\left(\mathrm{x}^{t e m p}\right)-\beta\left\|\mathrm{x}^{k+1}-\mathrm{x}^{t e m p}\right\|^{2},
$$

and that $\mathrm{x}^{k+1}$ is a critical point of $\mathrm{G}_{\text {cELo }}^{\mathcal{E}}$. Then $\mathrm{x}^{k+1}=\mathrm{x}^{\text {temp }}$ means that $\mathrm{x}^{\text {temp }}$ was a critical point of $\mathrm{G}_{\mathrm{CEL} O}$ and that the inner loop has been broken thanks to $\sigma_{k}^{-}=\emptyset$. In this case the algorithm stops. Otherwise, if $\mathrm{x}^{k+1} \neq \mathrm{x}^{\text {temp }}$ we get from (D.10),

$$
\mathrm{G}_{\text {cELo }}^{\mathcal{E}}\left(\mathrm{x}^{k+1}\right)<\mathrm{G}_{\text {cELO }}^{\mathcal{E}}\left(\mathrm{x}^{t e m p}\right)=\mathrm{G}_{\text {cELo }}^{\mathcal{E}}\left(\mathrm{x}^{k}\right),
$$

which means that the algorithm moves to another value $\mathrm{G}_{\text {CELO }}^{\mathcal{E}}\left(\mathrm{x}^{k+1}\right) \in \mathrm{G}_{\mathrm{CEL} 0}^{\mathcal{E}}(\mathcal{C})$ strictly lower than $\mathrm{G}_{\text {cELO }}^{\mathcal{E}}\left(\mathrm{x}^{k}\right)$. Moreover, for all global minimizer $\hat{\mathrm{x}} \in \mathcal{C}$ (there exists at least one from Proposition 4.6), Lemma 4.4 ensures that $\hat{\mathrm{x}}^{0}$ is also a global minimizer verifying $\sigma^{-}\left(\hat{\mathrm{x}}^{0}\right)=\emptyset$. Then there exists at least one element $\overline{\mathrm{x}} \in \mathcal{C}$ verifying $\sigma^{-}(\overline{\mathrm{x}})=\emptyset$ and $\mathrm{G}_{\text {cELo }}^{\mathcal{E}}(\overline{\mathrm{x}})=\inf \left\{\mathrm{G}_{\mathrm{CELO}}^{\mathcal{E}}(\mathcal{C})\right\}$. This last statement with (D.11) and the fact that $\mathrm{G}_{\mathrm{cELo}}^{\mathcal{E}}(\mathcal{C})$ is finite allow to conclude that there exists $k^{\star} \in \mathbb{N}$ such that $\sigma_{k^{\star}}^{-}=\emptyset$.

Finally, from Lemma 4.7 we get that $\mathrm{x}^{k^{\star}}$ is a (local) minimizer of $\mathrm{G}_{\ell_{0}}$. This completes the proof.

Acknowledgment The authors would like to thank the anonymous reviewers for their remarks and comments. In particular we thank Reviewer 2 for very detailed comments and suggestions helpful to strengthen this manuscript.

\section{References}

[1] Hedy Attouch, Jérôme Bolte, and Benar Fux Svaiter. Convergence of descent methods for semialgebraic and tame problems: proximal algorithms, forwardbackward splitting, and regularized gaussseidel methods. Mathematical Programming, 137(1-2):91-129, 2013. 
[2] Amir Beck and Marc Teboulle. A fast iterative shrinkage-thresholding algorithm for linear inverse problems. SIAM Journal on Imaging Sciences, 2(1):183-202, 2009.

[3] Andrew Blake and Andrew Zisserman. Visual reconstruction, volume 2. MIT press Cambridge, 1987.

[4] Thomas Blumensath and Mike E Davies. Iterative thresholding for sparse approximations. Journal of Fourier Analysis and Applications, 14(5-6):629-654, 2008.

[5] Jerôme Bolte, Aris Daniilidis, Adrian Lewis, and Masahiro Shiota. Clarke critical values of subanalytic lipschitz continuous functions. Ann. Polon. Math.(memorial issue for S. Lojasiewicz), 2005.

[6] Leo Breiman. Better subset regression using the nonnegative garrote. Technometrics, 37(4):373384, 1995.

[7] Emmanuel J Candès, Justin Romberg, and Terence Tao. Robust uncertainty principles: Exact signal reconstruction from highly incomplete frequency information. IEEE Transactions on Information Theory, 52(2):489-509, 2006.

[8] Emmanuel J Candes, Michael B Wakin, and Stephen P Boyd. Enhancing sparsity by reweighted $\ell_{1}$ minimization. Journal of Fourier analysis and applications, 14(5-6):877-905, 2008.

[9] Scott Shaobing Chen, David L Donoho, and Michael A Saunders. Atomic decomposition by basis pursuit. SIAM journal on scientific computing, 20(1):33-61, 1998.

[10] Sheng Chen, Colin FN Cowan, and Peter M Grant. Orthogonal least squares learning algorithm for radial basis function networks. IEEE Transactions on Neural Networks, 2(2):302-309, 1991.

[11] Frank H Clarke. Optimization and nonsmooth analysis, volume 5. SIAM, 1990.

[12] Patrick L Combettes and Valérie R Wajs. Signal recovery by proximal forward-backward splitting. Multiscale Modeling \& Simulation, 4(4):1168-1200, 2005.

[13] Ingrid Daubechies, Michel Defrise, and Christine De Mol. An iterative thresholding algorithm for linear inverse problems with a sparsity constraint. Communications on Pure and Applied Mathematics, 57(11):1413-1457, 2004.

[14] Geoff Davis, Stephane Mallat, and Marco Avellaneda. Adaptive greedy approximations. Constructive approximation, 13(1):57-98, 1997.

[15] Tao Pham Dinh and Hoai An Le Thi. Recent advances in dc programming and dca. In Transactions on Computational Intelligence XIII, pages 1-37. Springer, 2014.

[16] David L Donoho. For most large underdetermined systems of linear equations the minimal $\ell_{1}$ norm solution is also the sparsest solution. Communications on Pure and Applied Mathematics, 59(6):797-829, 2006.

[17] Jianqing Fan and Runze Li. Variable selection via nonconcave penalized likelihood and its oracle properties. Journal of the American Statistical Association, 96(456):1348-1360, 2001.

[18] Simon Foucart and Ming-Jun Lai. Sparsest solutions of underdetermined linear systems via $\ell_{q^{-}}$ minimization for $0<q \leq 1$. Applied and Computational Harmonic Analysis, 26(3):395-407, 2009 . 
[19] GM Fung and OL Mangasarian. Equivalence of minimal $\ell_{0^{-}}$and $\ell_{p^{-}}$norm solutions of linear equalities, inequalities and linear programs for sufficiently small p. Journal of optimization theory and applications, 151(1):1-10, 2011.

[20] Gilles Gasso, Alain Rakotomamonjy, and Stéphane Canu. Recovering sparse signals with a certain family of nonconvex penalties and DC programming. IEEE Transactions on Signal Processing, 57(12):4686-4698, 2009.

[21] Stuart Geman and Donald Geman. Stochastic relaxation, Gibbs distributions, and the Bayesian restoration of images. IEEE Transactions on Pattern Analysis and Machine Intelligence, (6):721741, 1984.

[22] Pinghua Gong, Changshui Zhang, Zhaosong Lu, Jianhua Huang, and Jieping Ye. A General Iterative Shrinkage and Thresholding Algorithm for Non-convex Regularized Optimization Problems. In Proceedings of The 30th International Conference on Machine Learning, pages 37-45, 2013.

[23] Matthieu Kowalski. Thresholding rules and iterative shrinkage/thresholding algorithm: A convergence study. In ICIP-International Conference on Image Processing, 2014.

[24] Hoai An Le Thi, Hoai Minh Le, and Tao Pham Dinh. Feature selection in machine learning: an exact penalty approach using a difference of convex function algorithm. Machine Learning, pages 1-24, 2014.

[25] Yvan G Leclerc. Constructing simple stable descriptions for image partitioning. International Journal of Computer Vision, 3(1):73-102, 1989.

[26] Stéphane G Mallat and Zhifeng Zhang. Matching pursuits with time-frequency dictionaries. IEEE Transactions on Signal Processing, 41(12):3397-3415, 1993.

[27] Hosein Mohimani, Massoud Babaie-Zadeh, Irina Gorodnitsky, and Christian Jutten. Sparse recovery using smoothed $\ell_{0}$ (SL0): Convergence analysis. arXiv preprint arXiv:1001.5073, 2010.

[28] Hosein Mohimani, Massoud Babaie-Zadeh, and Christian Jutten. A fast approach for overcomplete sparse decomposition based on smoothed $\ell_{0}$ norm. IEEE Transactions on Signal Processing, 57(1):289-301, 2009.

[29] Balas Kausik Natarajan. Sparse approximate solutions to linear systems. SIAM journal on computing, 24(2):227-234, 1995.

[30] Mila Nikolova. Markovian reconstruction using a gnc approach. IEEE Transactions on Image Processing, 8(9):1204-1220, 1999.

[31] Mila Nikolova. Analysis of the recovery of edges in images and signals by minimizing nonconvex regularized least-squares. SIAM Journal on Multiscale Modeling \& Simulation, 4(3):960-991, 2005 .

[32] Mila Nikolova. Description of the minimizers of least squares regularized with $\ell_{0}$-norm. Uniqueness of the global minimizer. SIAM Journal on Imaging Sciences, 6(2):904-937, 2013.

[33] Mila Nikolova. Relationship between the optimal solutions of least squares regularized with $\ell_{0^{-}}$ norm and constrained by k-sparsity. hal-00944006v1 Preprint, 2014.

[34] P. Ochs, A. Dosovitskiy, T. Brox, and T. Pock. On Iteratively Reweighted Algorithms for Nonsmooth Nonconvex Optimization in Computer Vision. SIAM Journal on Imaging Sciences, $8(1): 331-372,2015$. 
[35] Yagyensh Chandra Pati, Ramin Rezaiifar, and PS Krishnaprasad. Orthogonal matching pursuit: Recursive function approximation with applications to wavelet decomposition. In Signals, Systems and Computers, 1993. 1993 Conference Record of The Twenty-Seventh Asilomar Conference on, pages 40-44. IEEE, 1993.

[36] Dori Peleg and Ron Meir. A bilinear formulation for vector sparsity optimization. Signal Processing, 88(2):375-389, 2008.

[37] Marc C Robini, Aimé Lachal, and Isabelle E Magnin. A stochastic continuation approach to piecewise constant reconstruction. IEEE Transactions on Image Processing, 16(10):2576-2589, 2007.

[38] Marc C Robini and Isabelle E Magnin. Optimization by stochastic continuation. SIAM journal on Imaging Sciences, 3(4):1096-1121, 2010.

[39] Charles Soussen, Jérôme Idier, David Brie, and Junbo Duan. From Bernoulli-Gaussian deconvolution to sparse signal restoration. IEEE Transactions on Signal Processing, 59(10):4572-4584, 2011.

[40] Vladimir N Temlyakov. Greedy approximation. Acta Numerica, 17:235-409, 2008.

[41] Joel A Tropp. Greed is good: Algorithmic results for sparse approximation. IEEE Transactions on Information Theory, 50(10):2231-2242, 2004.

[42] Cun-Hui Zhang. Nearly unbiased variable selection under minimax concave penalty. The Annals of Statistics, pages 894-942, 2010.

[43] Hui Zou. The adaptive lasso and its oracle properties. Journal of the American statistical association, 101(476):1418-1429, 2006. 